\title{
Highly interpretable hierarchical deep rule-based classifier
}

Xiaowei $\mathrm{Gu}^{\mathrm{a}, \mathrm{b}, *, 1}$

x.gu3@lancaster.ac.uk

Plamen P. Angelov ${ }^{\mathrm{a}, \mathrm{b}, \mathrm{c}, 1}$

p.angelov@lancaster.ac.uk

aSchool of Computing and Communications, Lancaster University, Lancaster, LA1 4WA, UK

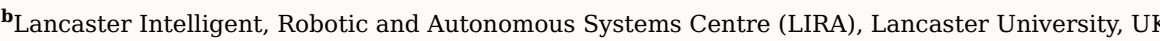

'Honorary Professor at Technical University, Sofia, 1000, Bulgaria

${ }^{*}$ Corresponding author at: School of Computing and Communications, Lancaster University, Lancaster, LA1 4WA, UK.

${ }^{1} \mathrm{X} . \mathrm{Gu}$ and P. Angelov contributed equally.

Abstract

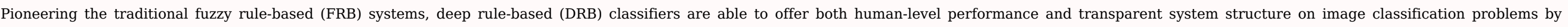

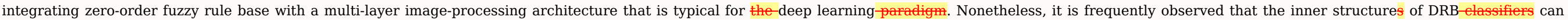

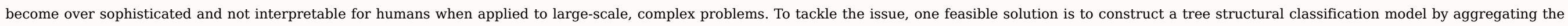

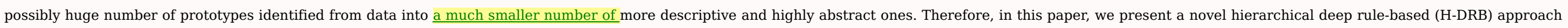

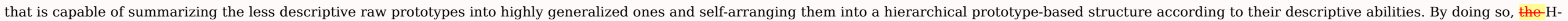

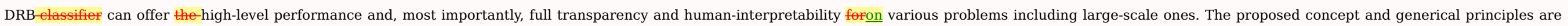
verified through numerical experiments based on a wide variety of popular benchmark image sets. Numerical results demonstrate that the promise of the-H-DRB-approach.

Keywords: Deep rule-based; Hierarchical; Prototype-based; Self-organizing

\section{Introduction}

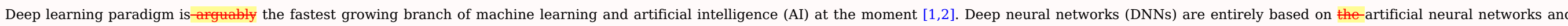

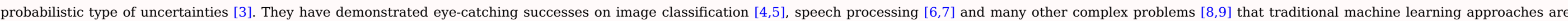
struggling with

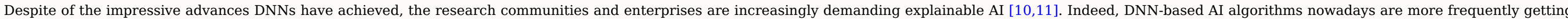

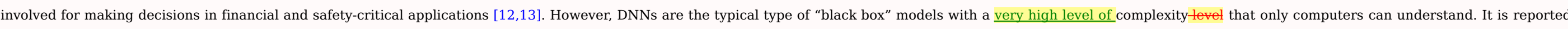

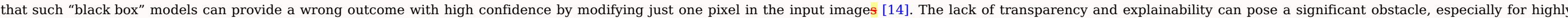

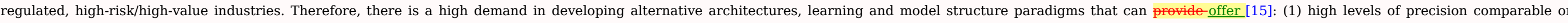

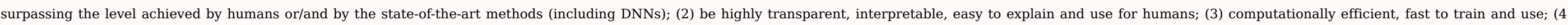
computational resource and training data lean - able to be trained with a single or handful or examples per class, not requiring computer accelerators, such as GPU, HPC, etc.

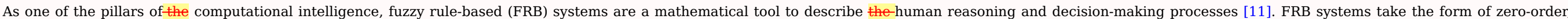




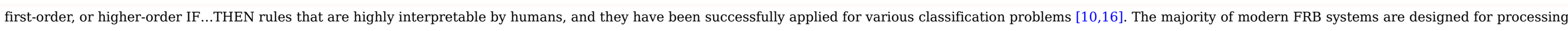

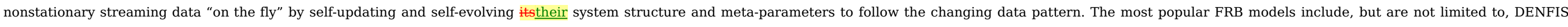

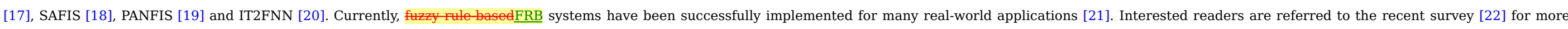

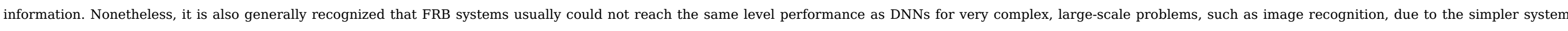
structure and operating mechanism than DNNs.

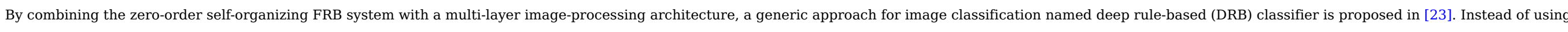

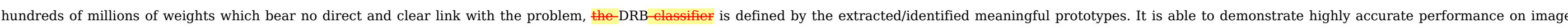

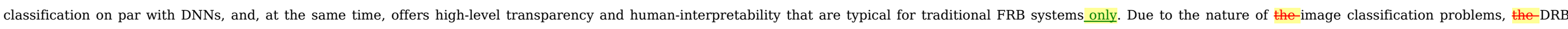

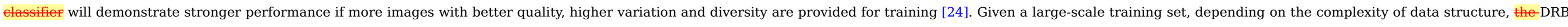

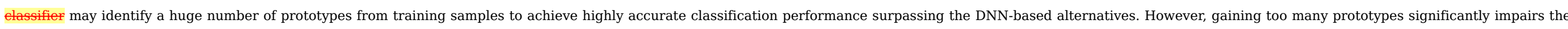

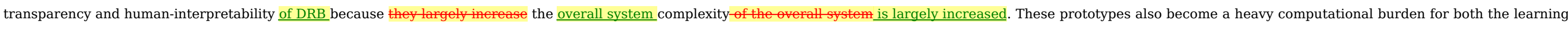
and decision-making processes of the classifier because each training/validation image will be compared with all identified prototypes in terms of their visual similarity

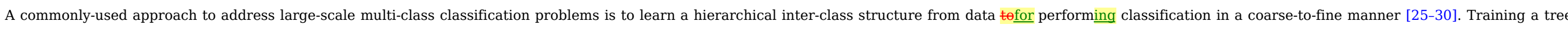

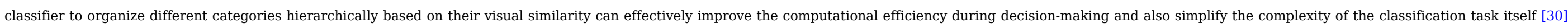

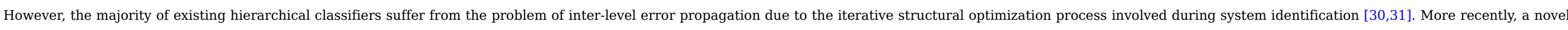

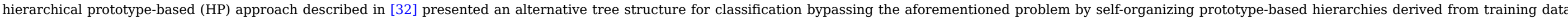

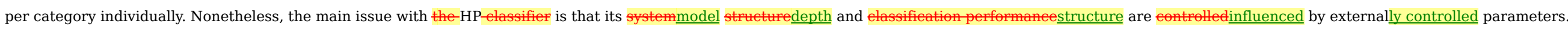

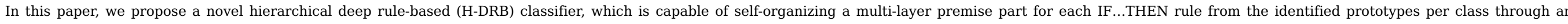

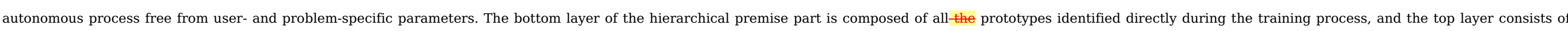

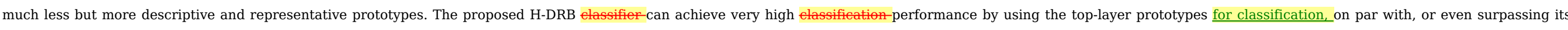

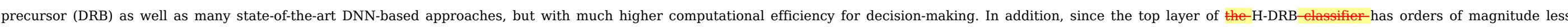

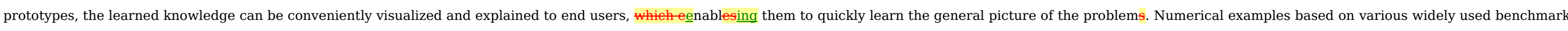
image data-sets are performed to demonstrate the effectiveness and validity of the proposed concept and general principles.

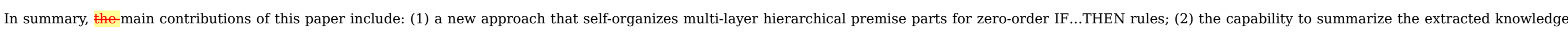

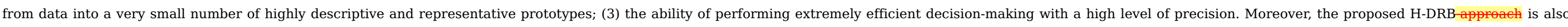
free from externally controlled parameters, prior assumptions on data generation model as well as the problem of inter-level error propagation.

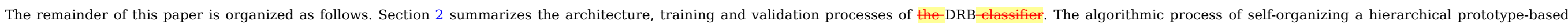
structure is presented in Section 3. Numerical examples are given in Section 4, and this paper is concluded by Section 5.

\section{Deep rule-based classifier}

In this section, the general architecture, algorithmic procedures of the-DRB-classifier are briefly described to make this paper self-contained. Key notations are summarized in Table 1 for clarity.

Table 1 Definitions of key notations used in this paper.

Notations $\quad$ Definitions

Number of image classes

A particular image 


\begin{tabular}{|c|c|}
\hline $\boldsymbol{x}$ & Feature vector of $\mathbf{I}$ \\
\hline$D$ & Data density \\
\hline$D^{M}$ & Multimodal data density \\
\hline $\mathrm{F}(\mathbf{I})$ & Discriminative representation extracted from $\mathbf{I}$ by the feature descriptor \\
\hline $\mathbf{R}^{M}$ & Data space \\
\hline M & Dimensionality of the data space \\
\hline$N_{i}$ & Number of prototypes of the $i$ th class \\
\hline$\lambda_{i}$ & Degree of visual similarity \\
\hline$K_{i}$ & Number of processed training images of the $i$ th class \\
\hline $\boldsymbol{\mu}_{i}$ & Global mean of feature vectors of training images of the $i$ th class. \\
\hline$\{\mathbf{P}\}_{i}$ & Raw prototypes of the $i$ th class \\
\hline$\{\boldsymbol{p}\}_{i}$ & Feature vectors of $\{\mathbf{P}\}_{i}$ \\
\hline $\mathbf{P}_{i, j}$ & The $j$ th raw prototype of the $i$ th class \\
\hline $\boldsymbol{p}_{i, j}$ & Feature vector of $\mathbf{P}_{i, j}$ \\
\hline$S_{i, j}$ & Cardinality of $\mathbf{P}_{i, j}$ \\
\hline$r_{i, j}$ & Radius of the influential arearea of influence of $\mathbf{P}_{i, j}$ \\
\hline$\{\mathbf{P}\}_{i}^{t}$ & Highly descriptive prototypes of the $i$ th class obtained after the $t^{\text {th }}$ filtering round \\
\hline$\{\boldsymbol{p}\}_{i}^{t}$ & Feature vectors of $\{\mathbf{P}\}_{i}^{t}$ \\
\hline $\mathbf{P}_{i, j}^{t}$ & The $j$ th highly descriptive prototype of the $i$ th class obtained after the $t^{t h}$ filtering round \\
\hline $\boldsymbol{p}_{i, j}^{t}$ & Feature vector of $\mathbf{P}_{i, j}^{t}$ \\
\hline $\mathbb{C}_{i, j}^{t}$ & Cluster formed around $\boldsymbol{p}_{i, j}^{t}$ \\
\hline$S_{i, j}^{t}$ & Cardinality of $\mathbb{C}_{i, j}^{t}$ \\
\hline$T_{i}$ & Number of filtering rounds before the algorithm converges \\
\hline
\end{tabular}




\subsection{General architecture}

The general architecture of a DRB classifier is given by Fig. 1 [23]. As we can see from this figure, athe typical DRB-elassifier is composed of the following four components:

(1) Pre-processing module.

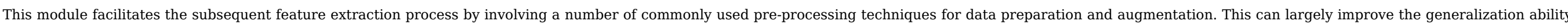

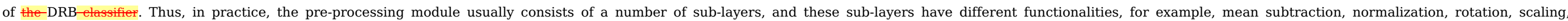
segmentation [23].

(2) Feature descriptor.

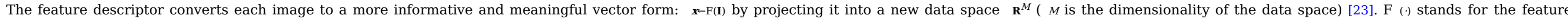

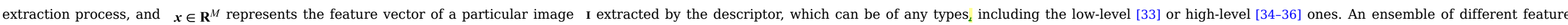
descriptors ean-may be created to further improve the descriptive ability.

(3) Massively parallel rule base.

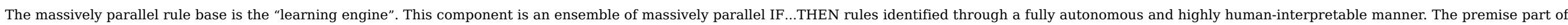
each IF...THEN rule is composed of a (possibly huge) number of prototypes identified from the training images of a particular class (thus, one rule per class), and is formulated as [23]:

$\operatorname{IF}\left(\mathbf{I} \sim \mathbf{P}_{i, 1}\right) \operatorname{OR}\left(\mathbf{I} \sim \mathbf{P}_{i, 2}\right)$ OR $\ldots$ OR $\left(\mathbf{I} \sim \mathbf{P}_{i, N_{i}}\right) \quad \operatorname{THEN}($ Class $i)$,

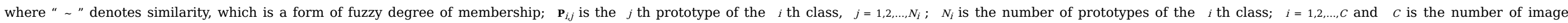
elassescategories in the training set.

(4) Decision-maker

This component determines the semantic label of each validation image.

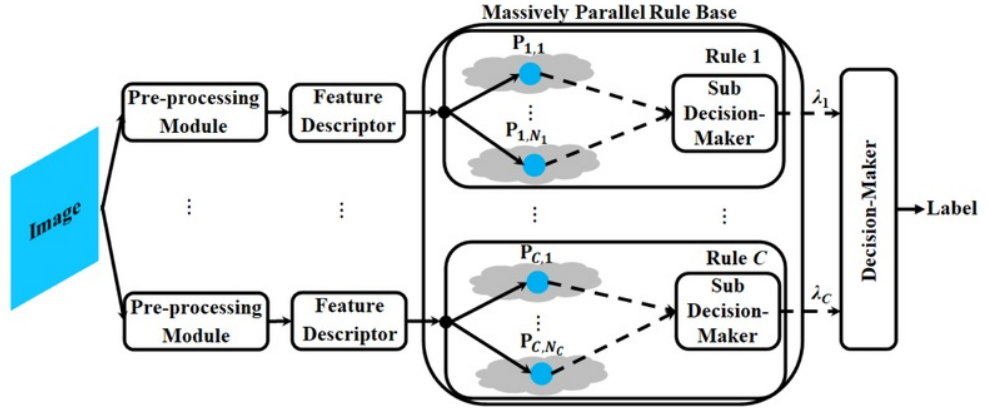

\subsection{System identification process}

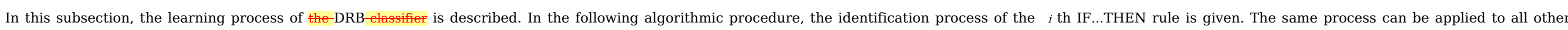
IF...THEN rules within the same rule base [23]. 


\section{The identification process of the $i^{\text {th }}$ IF...THEN rule:}

Step 1. The feature vector of the current image of the $i$ th class, $\mathbf{I}_{i, K_{i}}$ is extracted and normalized by the its $L_{2}$ norm:

$$
\boldsymbol{x}_{i, K_{i}} \leftarrow \frac{\mathrm{F}\left(\mathbf{I}_{i, K_{i}}\right)}{\left\|\mathrm{F}\left(\mathbf{I}_{i, K_{i}}\right)\right\|},
$$

where $\|x\|=\sqrt{\boldsymbol{x}^{T} \boldsymbol{x}}$

If this is the first image, namely, $K_{i}=1$, the global meta-parameters of the $i$ th IF...THEN rule are initialized by

$$
N_{i} \leftarrow 1 ; \quad \mu_{i} \leftarrow x_{i, K_{i}}
$$

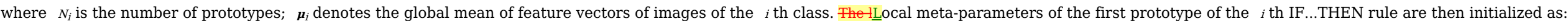

$\mathbf{P}_{i, N_{i}} \leftarrow \mathbf{I}_{i, K_{i}} ; \quad \boldsymbol{p}_{i, N_{i}} \leftarrow \boldsymbol{x}_{i, K_{i}} ; \quad S_{i, N_{i}} \leftarrow 1 ; \quad r_{i, N_{i}} \leftarrow r_{o}$

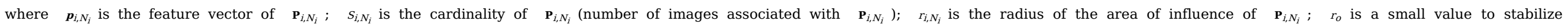
$r_{o}=\sqrt{2\left(1-\cos \left(\frac{\pi}{6}\right)\right)} \cdot$ At the end, the $i$ th IF...THEN rule is initialized as:

$\operatorname{IF}\left(\mathbf{I} \sim \mathbf{P}_{i, N_{i}}\right) \quad \operatorname{THEN}($ Class $i)$.

Otherwise (namely, $K_{i} \geq 1$ ), the global mean $\boldsymbol{\mu}_{i}$ is updated by $\boldsymbol{x}_{i, K_{i}}$ :

$\boldsymbol{\mu}_{i} \leftarrow \frac{K_{i}-1}{K_{i}} \boldsymbol{\mu}_{i}+\frac{1}{K_{i}} \boldsymbol{x}_{i, K_{i}}$

Step 2. The dData densitiesy values at $\mathbf{I}_{i, K_{i}}$ and $\mathbf{P}_{i, 1}, \mathbf{P}_{i, 2}, \ldots, \mathbf{P}_{i, N_{i}}$ are calculated by Eq. (7):

$$
D(\mathbf{Z})=\frac{1}{1+\frac{\left\|z-\boldsymbol{\mu}_{i}\right\|^{2}}{1-\left\|\boldsymbol{\mu}_{i}\right\|^{2}}},
$$

where $\quad \mathbf{Z}=\mathbf{I}_{i, K_{i}}, \mathbf{P}_{i, 1}, \mathbf{P}_{i, 2}, \ldots, \mathbf{P}_{i, N_{i}} ; \quad z=\boldsymbol{x}_{i, K_{i}}, \boldsymbol{p}_{i, 1}, \boldsymbol{p}_{i, 2}, \ldots, \boldsymbol{p}_{i, N_{i}}$.

The nearest prototype, $\mathbf{P}_{i, n^{*}}$ to $\mathbf{I}_{i, K_{i}}$ is identified by Eq. (8):

$$
n^{*}=\min _{j=1,2, \ldots, N_{i}}\left(\left\|\boldsymbol{p}_{i, j}-\boldsymbol{x}_{i, K_{i}}\right\|\right)
$$

Step 3. Condition 1 is examined firstly to see whether $\mathbf{I}_{i, K_{i}}$ can be a new prototype

Condition 1:

$$
\begin{gathered}
\text { If }\left(D\left(\mathbf{I}_{i, K_{i}}\right)>\max _{j=1,2, \ldots, N_{i}}\left(D\left(\mathbf{P}_{i, j}\right)\right)\right) \\
\operatorname{Or}\left(D\left(\mathbf{I}_{i, K_{i}}\right)<\min _{j=1,2, \ldots, N_{i}}\left(D\left(\mathbf{P}_{i, j}\right)\right)\right) \\
\text { Or }\left(\left\|\boldsymbol{x}_{i, K_{i}}-\boldsymbol{p}_{i, n^{*}}\right\| \geq r_{i, n^{*}}\right) \\
\text { Then }\left(\mathbf{I}_{i, K_{i}} \text { is a new prototype }\right)
\end{gathered}
$$

If Condition 1 is met, a new prototype is added by Eq. (10):

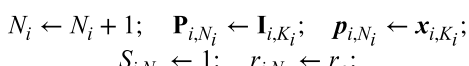


Otherwise, the lelocal meta-parameters of the nearest prototype are updated:

$\boldsymbol{p}_{i, n^{*}} \quad \leftarrow \frac{S_{i, n^{*}}}{S_{i, n^{*}+1}} \boldsymbol{p}_{i, n^{*}}+\frac{1}{S_{i, n^{*}+1}} \boldsymbol{x}_{i, K_{i}} ;$

$r_{i, n^{*}} \leftarrow \frac{1}{2} \sqrt{S_{i, n^{*}} \leftarrow S_{i, n^{*}}^{2}+1}$

Then, the algorithm goes back to Step $\mathbf{1}$ if new images are available.

\subsection{Validation process}

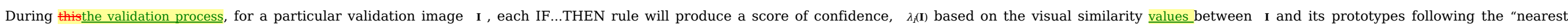
prototype" principle:

$$
\lambda_{i}(\mathbf{I})=\max _{j=1,2, \ldots, N_{i}}\left(\lambda_{i j}(\mathbf{I})\right)=\max _{j=1,2, \ldots, N_{i}}\left(e^{-\left\|x-p_{i j}\right\|^{2}}\right),
$$

The decision maker, then, determines the label of $\mathbf{I}$ based on the $c$ scores of confidence (namely, $\lambda_{1}(\mathbf{I}), \lambda_{2}(\mathbf{I}), \ldots, \lambda_{C}(\mathbf{I})$ ) following the "winner takes all" principle:

Label $(\mathbf{I}) \leftarrow$ Class $^{*} ; \quad i^{*}=\underset{i=1,2, \ldots, C}{\operatorname{argmax}}\left(\lambda_{i}(\mathbf{I})\right)$

\section{Self-organizing the premise part into a hierarchical form}

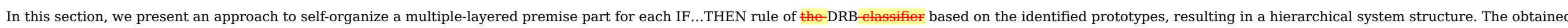
model is renamed as the hierarchical DRB (H-DRB) classifier.

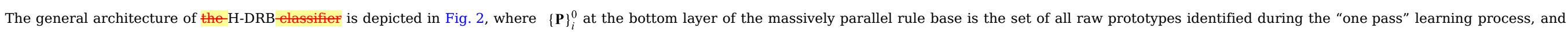
$\{\mathbf{P}\}_{i}^{T_{i}}$ is a set of highly descriptive prototypes at the top layer; $\{\mathbf{P}\}_{i}^{1},\{\mathbf{P}\}_{i}^{2}, \ldots, \quad\{\mathbf{P}\}_{i}^{T_{i}-1}$ are the sets of prototypes at the first, second, last hidden layers.

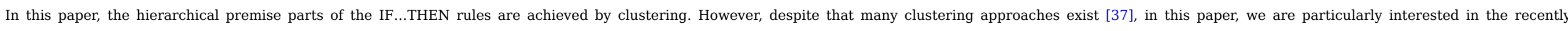

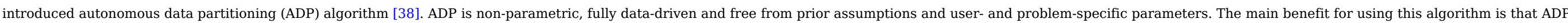

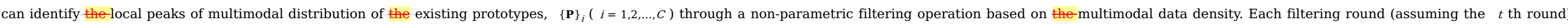

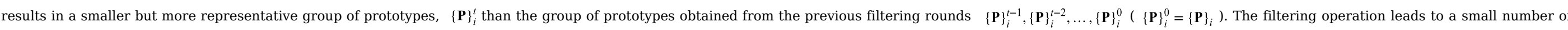

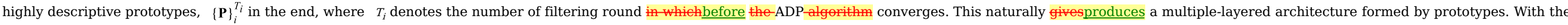

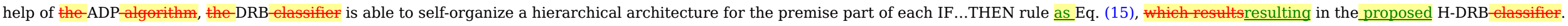

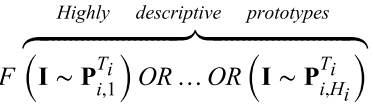

$$
\begin{aligned}
& \text { Prototypes connected to } \mathbf{P}_{i, 1}^{T_{i}} \\
& \text { THENIF } \overbrace{\left(\mathbf{I} \sim \mathbf{P}_{i, 1}\right) \text { OR ..OR }\left(\mathbf{I} \sim \mathbf{P}_{i, m}\right)} \text { OR. } \\
& \text { Prototypes connected to } \mathbf{P}_{i, H_{i}}^{T_{i}} \\
& \text { OR } \overbrace{\left(\mathbf{I} \sim \mathbf{P}_{i, n}\right) \text { OR } \ldots \text { OR }\left(\mathbf{I} \sim \mathbf{P}_{i, N_{i}}\right)} \\
& \text { THEN(Class } i) \text {, }
\end{aligned}
$$


where $\quad \mathbf{P}_{i, j}^{T_{i}} \in\{\mathbf{P}\}_{i}^{T_{i}} ; \quad H_{i}$ is the number of prototypes at the top layer; $\mathbf{P}_{i, 1}, \ldots, \mathbf{P}_{i, m} \in\{\mathbf{P}\}_{i}$ are the prototypes at the bottom layer of the hierarchical architecture that are connected to $\mathbf{P}_{i, 1}^{T_{i}}$ through the hidden layers;

$\mathbf{P}_{i, n}, \ldots, \mathbf{P}_{i, N_{i}} \in\{\mathbf{P}\}_{i}$ are the prototypes connected to $\mathbf{P}_{i, H_{i}}^{T_{i}}$.

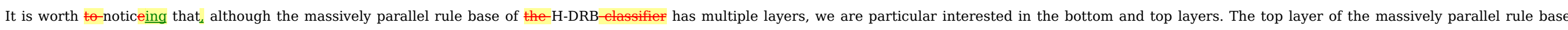

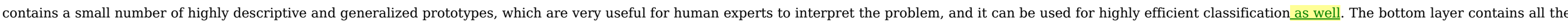

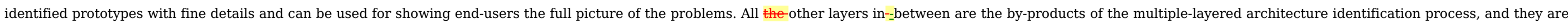
less important compared with the bottom and top ones. Therefore, they can be viewed as hidden layers. By default, the-H-DRB-classifier will use the top layer for decision-making with Eqs. (13) and (14).

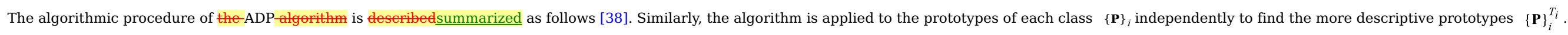

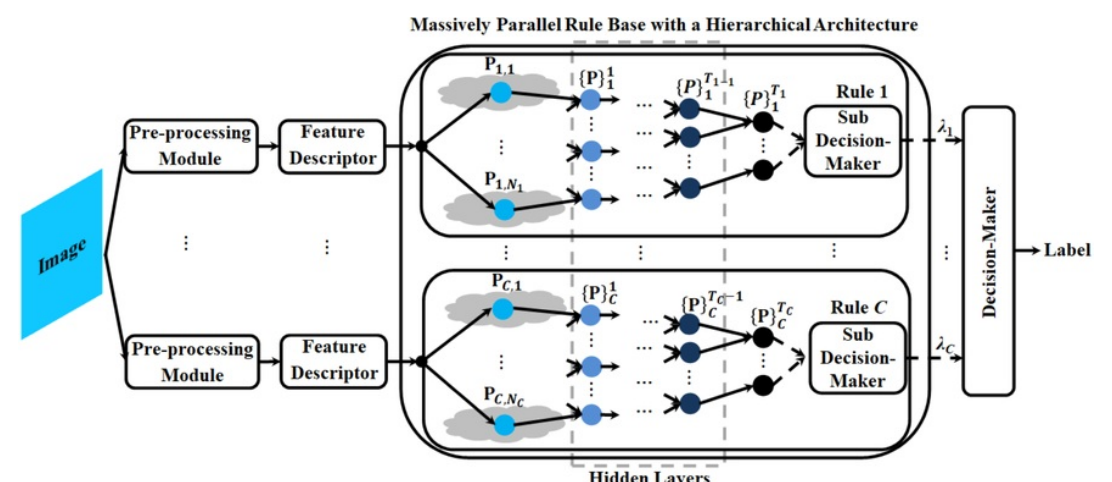

Fig. 2 General architecture of H-DRB-classifiers.

\section{The identification process of the highly descriptive prototypes of the $i^{\text {th }}$ class:}

Step 1. Firstly, data densities of the feature vectors, denoted by $\{\boldsymbol{p}\}_{i}$ of the identified prototypes $\{\mathbf{P}\}_{i}$ in the feature space are calculated by Eq. (16):

$D\left(\boldsymbol{p}_{i, j}\right)=\frac{1}{1+\frac{\left\|\boldsymbol{p}_{i, j}-\boldsymbol{\rho}_{\boldsymbol{\rho}}\right\|^{2}}{1-\left\|\boldsymbol{\rho}_{i}\right\|^{2}}}$,

where $\boldsymbol{\rho}_{i}=\frac{1}{N_{i}} \sum_{l=1}^{N_{i}} \boldsymbol{p}_{i, l} ; j=1,2, \ldots, N_{i}$.

The feature vector with the highest data density value (re-denoted by $z_{i, 1}$ ) is identified using Eq. (17):

$\boldsymbol{z}_{i, 1} \leftarrow \boldsymbol{p}_{i, m^{*}} ; \quad m^{*}=\underset{j=1,2, \ldots, N_{i}}{\operatorname{argmax}}\left(D_{K_{i}}\left(\boldsymbol{p}_{i, j}\right)\right)$,

and $\boldsymbol{p}_{i, m^{*}}$ is removed from $\{\boldsymbol{p}\}_{i}$, namely, $\{\boldsymbol{p}\}_{i} \leftarrow\{\boldsymbol{p}\}_{i} \backslash \boldsymbol{p}_{i, m^{*}}$.

Then, the feature vector nearest to $\boldsymbol{z}_{i, 1}$ is identified and removed from $\{\boldsymbol{p}\}_{i}$ :

$\boldsymbol{z}_{i, 2} \leftarrow \boldsymbol{p}_{i, n^{*}} ; \quad \boldsymbol{p}_{i, n^{*}}=\underset{\boldsymbol{q} \in\{\boldsymbol{p}\}_{i}}{\operatorname{argmin}}\left(\left\|z_{i, 1}-\boldsymbol{q}\right\|\right)$,

and, $\boldsymbol{z}_{i, 3}$ can be identified by finding the feature vector that is closet to $\boldsymbol{z}_{i, 2}$. By repeating the same process, one can obtain a rank-ordered sequence, namely, $\left\{\boldsymbol{z}_{i, 1}, \boldsymbol{z}_{i, 2}, \boldsymbol{z}_{i, 3}, \ldots, \boldsymbol{z}_{i, N_{i}}\right\}$

Step 2. The 1 Local maxima of the-data density, denoted by $\{\boldsymbol{p}\}_{i}^{t}(t=1)$ are identified from $\left\{\boldsymbol{z}_{i, 1}, \boldsymbol{z}_{i, 2}, \boldsymbol{z}_{i, 3}, \ldots, \boldsymbol{z}_{i, N_{i}}\right\}$ by Condition 2 :

Condition 2: 


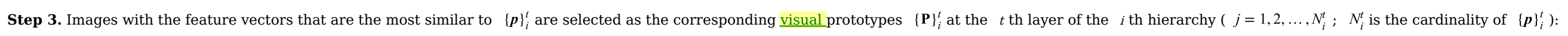

$\mathbf{P}_{i, j}^{t} \leftarrow \mathbf{I}_{i, n^{*}} ; \quad n^{*}=\underset{k=1,2, \ldots, K_{i}}{\operatorname{argmin}}\left(\left\|\boldsymbol{p}_{i, j}^{t}-\boldsymbol{x}_{i, k}\right\|\right)$.

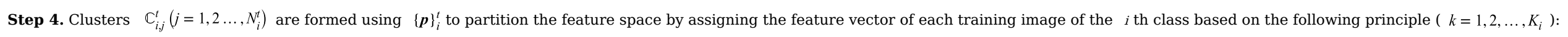

$\mathbb{C}_{i, n^{*}}^{t} \leftarrow \mathbb{C}_{i, n^{*}}^{t} \cup\left\{\boldsymbol{x}_{i, k}\right\} ; \quad n^{*}=\underset{j=1,2 \ldots, N_{i}^{t}}{\operatorname{argmin}}\left(\left\|\boldsymbol{p}_{i, j}^{t}-\boldsymbol{x}_{i, k}\right\|\right)$.

Then, the multimodal data density at the centre of each cluster is calculated as follows:

$$
D^{M}\left(\boldsymbol{\rho}_{i, j}^{t}\right)=\frac{S_{i, j}^{t}}{1+\frac{\left\|\boldsymbol{\rho}_{i, j}^{t}-\boldsymbol{\rho}_{i}\right\|^{2}}{1-\left\|\boldsymbol{\rho}_{i}\right\|^{2}}},
$$

where $\boldsymbol{\rho}_{i, j}^{t}=\frac{1}{S_{i, j}^{t}} \sum_{\mathbf{P} \in \mathbb{C}_{i, n^{*}}} \boldsymbol{p} ; S_{i, j}^{t}$ is the cardinality of $\mathbb{C}_{i, j}^{t}$

Step 5. The data-driven threshold, $\chi_{i}^{t}$ that defines the radius of neighbouring area around each cluster centre is derived from data by Eq. (23):

$$
\begin{gathered}
\eta_{i}^{t}=\frac{\sum_{p=1}^{N_{i}^{t}-1} \Sigma_{q=p_{i}}^{N_{i}^{t}}\left\|\boldsymbol{\rho}_{i, p}^{t}-\boldsymbol{\rho}_{i, q}^{t}\right\|}{N_{i}^{t}\left(N_{i}^{t}-1\right)} ; \\
\gamma_{i}^{t}=\frac{\sum_{\boldsymbol{x} \boldsymbol{y} \in\{\rho\}_{i}^{t} \boldsymbol{x} \boldsymbol{x} \boldsymbol{y}\|\boldsymbol{x}-\boldsymbol{y}\| \leq \eta_{i}^{t}}\|\boldsymbol{x}-\boldsymbol{y}\|}{M_{\eta_{i}^{t}}} ; \\
\chi_{i}^{t}=\frac{\sum_{\boldsymbol{x} \boldsymbol{y} \in\{\rho\}_{i}^{t} \boldsymbol{x} \neq \boldsymbol{y},\|\boldsymbol{x}-\boldsymbol{y}\| \leq \gamma_{i}^{t}}\|\boldsymbol{x}-\boldsymbol{y}\|}{M_{\gamma_{i}^{t}}}, \\
\text { where }\{\boldsymbol{\rho}\}_{i}^{t}=\left\{\boldsymbol{\rho}_{i, 1}^{t}, \boldsymbol{\rho}_{i, 2}^{t}, \ldots, \boldsymbol{\rho}_{i, N_{i}^{t}}^{t}\right\} .
\end{gathered}
$$

Then, for each cluster, $\mathbb{C}_{i, j}^{t}$, the collection of its neighbouring clusters, denoted by $\{\mathbb{C}\}_{i, j}^{n^{*}}$ are $\underline{\text { is }}$ identified by Condition 3:

\section{Condition 3:}

If $\left(\left\|\boldsymbol{\rho}_{i, j}^{t}-\boldsymbol{\rho}_{i, k}^{t}\right\| \leq \chi_{i}^{t}\right) \quad$ Then $\left(\mathbb{C}_{i, k}^{t} \in\{\mathbb{C}\}_{i, j}^{n^{*}}\right)$,

where $j, k=1,2, \ldots, N_{i}^{t} ; j \neq k$.

The leLocal maxima of multimodal data density are identified by Condition 4, which are denoted by $\{\boldsymbol{p}\}_{i}^{t+1}$ :

\section{Condition 4:}

$$
\operatorname{If}\left(D^{M}\left(\mathbb{C}_{i, j}^{t}\right)>\max _{\mathbb{C} \in\{\mathbb{C}\}_{i j}^{n^{*}}}\left(D^{M}(\mathbb{C})\right)\right) \quad \text { Then }\left(\boldsymbol{\rho}_{i, j}^{t} \in\{\boldsymbol{p}\}_{i}^{t+1}\right),
$$

where $j=1,2, \ldots, N_{i}^{t}$. After $\{\boldsymbol{p}\}_{i}^{t+1}$ is identified, the algorithm goes back to Step 3 and begins a new iteration $(t \leftarrow t+1)$ until the protypes do not change any morethe algorithm converges.

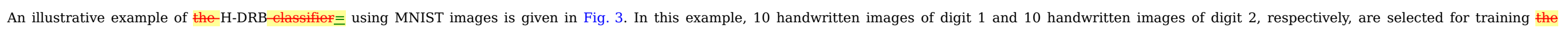

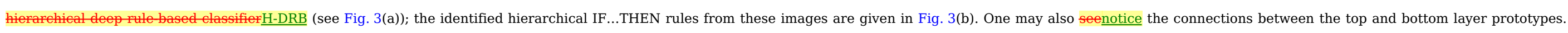

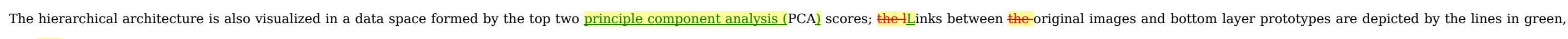
and the-links between the top and bottom layer prototypes are given by the lines in yellow. 
(a) Digits for training

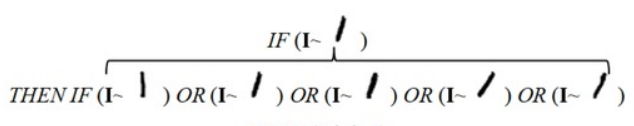

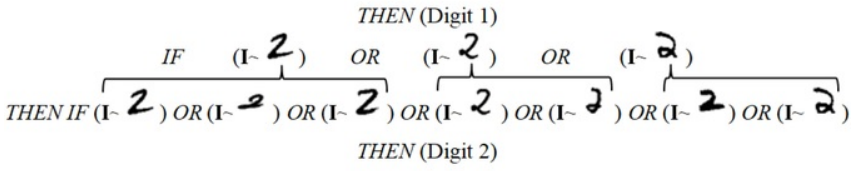

(b) The identified hierarchical IF...THEN rules

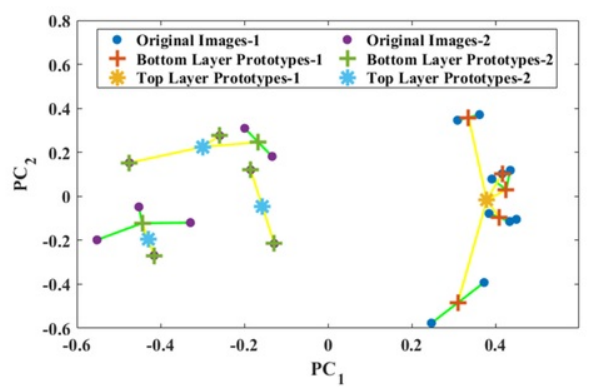

(c) Visualization of the hierarchical architecture using PCA scores

Fig. 3 Illustrative example using MNIST images.

\section{Numerical experiments and discussions}

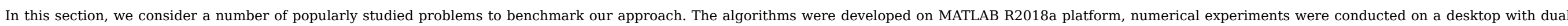
core processor $3.60 \mathrm{GHz} \times 2$ and $16 \mathrm{~GB}$ RAM.

\subsection{Dataset descriptions and experimental settings}

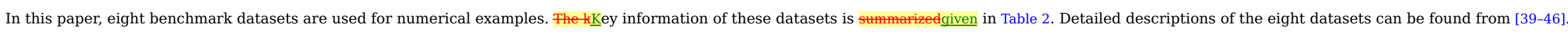

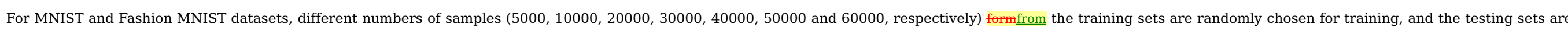

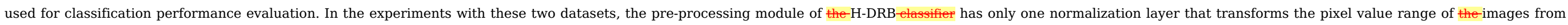

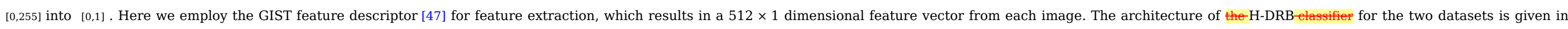
Fig. 4(a).

Table 2 Key information of the eight benchmark datasets.

\begin{tabular}{|c|c|c|c|c|c|}
\hline \multicolumn{2}{|c|}{ Dataset } & \# Images & \# Class & \# Images Per Class & \# Pixels \\
\hline \multirow[t]{2}{*}{ MNIST [39] } & Training set & 60000 & \multirow[t]{2}{*}{10} & $\sim 6000$ & \multirow[t]{2}{*}{$28 \times 28$} \\
\hline & Testing set & 10000 & & $\sim 1000$ & \\
\hline
\end{tabular}




\begin{tabular}{|c|c|c|c|c|c|}
\hline \multirow[t]{2}{*}{ Fashion MNIST [40] } & Training Set & 60000 & & 6000 & \\
\hline & Testing set & 10000 & & 1000 & \\
\hline \multicolumn{2}{|l|}{ RSSCN7 [42] } & 2800 & 7 & 400 & $400 \times 400$ \\
\hline \multicolumn{2}{|l|}{ Singapore [41] } & 1086 & 9 & $42-179$ & \multirow[t]{2}{*}{$256 \times 256$} \\
\hline \multicolumn{2}{|l|}{ UCMerced [43] } & 2100 & 21 & 100 & \\
\hline \multicolumn{2}{|l|}{ WuHan-RS19 [44] } & 950 & 19 & 50 & $600 \times 600$ \\
\hline \multicolumn{2}{|l|}{ Caltech101 [45] } & 8677 & 101 & $40-800$ & \multirow[t]{2}{*}{$\sim 200 \times 300$} \\
\hline \multicolumn{2}{|l|}{ Caltech256 [46] } & 29780 & 256 & $80-800$ & \\
\hline
\end{tabular}

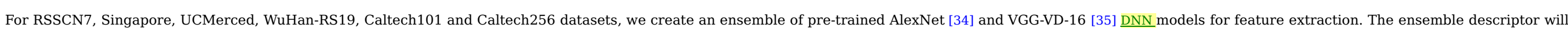
extract a highly discriminative representation from each training/testing image, I as:

$\boldsymbol{x} \leftarrow \mathrm{F}(\mathbf{I})=\left[\frac{\mathrm{AN}(\mathbf{I})}{\|\mathrm{AN}(\mathbf{I})\|}, \frac{\mathrm{VN}(\mathbf{I})}{\|\mathrm{VN}(\mathbf{I})\|}\right]^{T}$

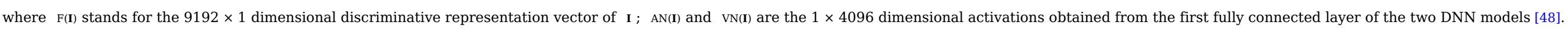

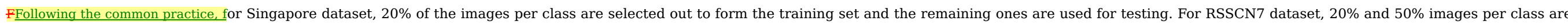

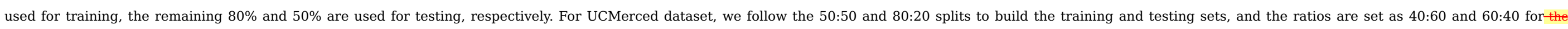

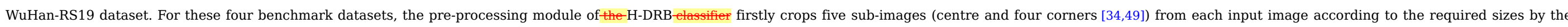

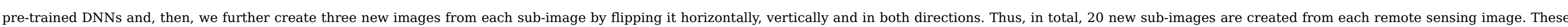

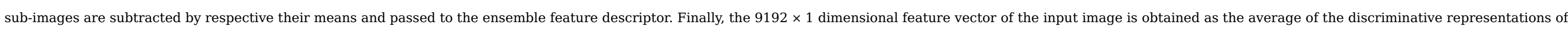
the 20 sub-images. The architecture for the four remote sensing problems is given in Fig. 4(b).

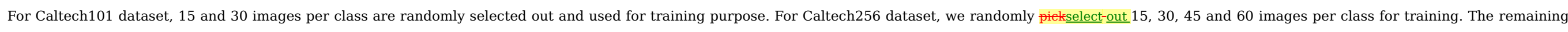

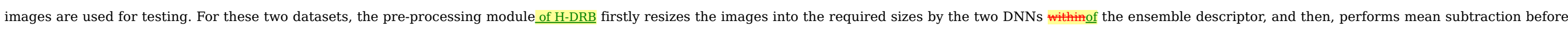
feature extraction. The architecture of H-DRB-lassifier for Caltech datasets is given in Fig. 4(c).

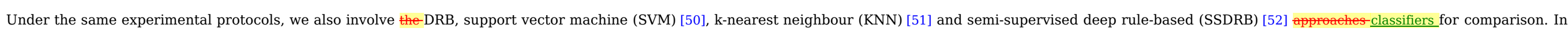

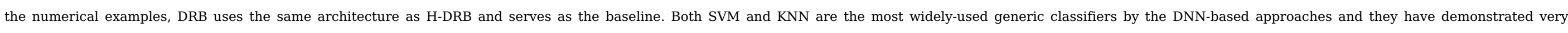

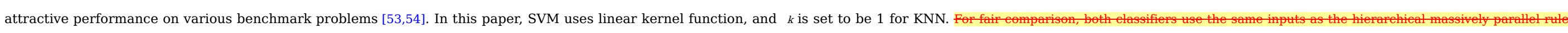

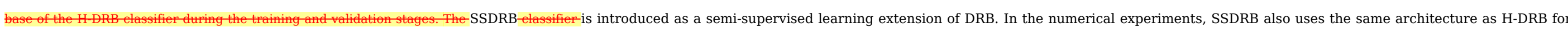

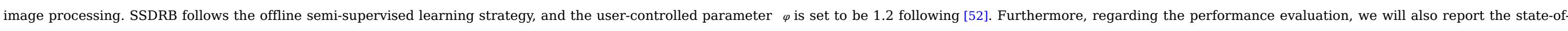

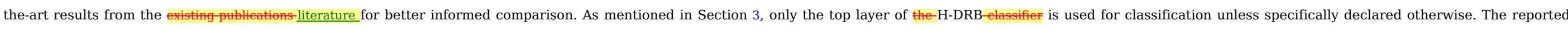

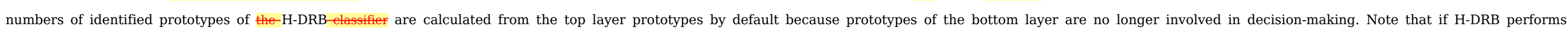
classification with its bottom layer prototypes, the results will be exactly the same as DRB 


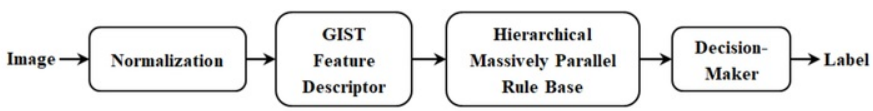

(a) The architecture for MNIST and Fashion MNIST datasets

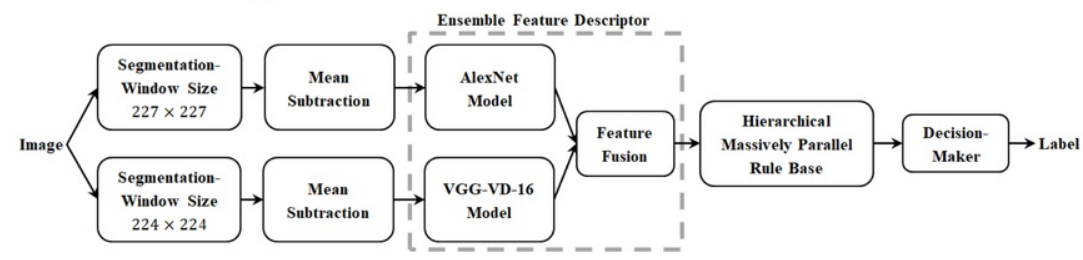

(b) The architecture for remote sensing datasets

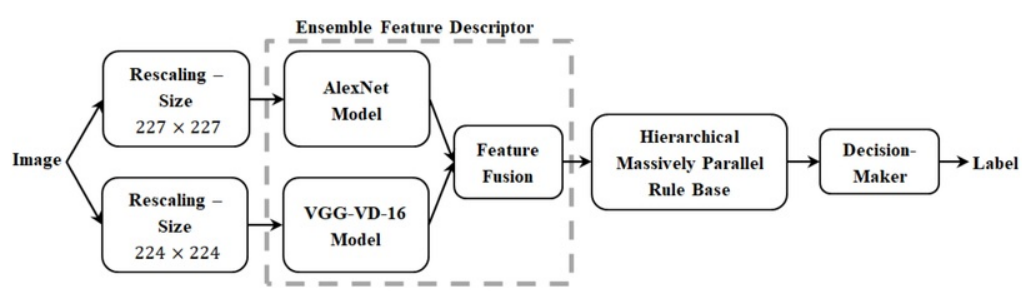

(c) The architecture for Caltech datasets

Fig. 4 Architectures of the-H-DRB

\subsection{Numerical results}

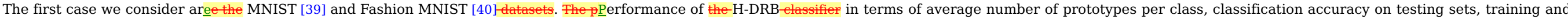
testing time consumption (both in seconds) on the two datasets are tabulated in Table 3, respectively. The $p$ Performance of the-DRB-elassifier is also reported as the baseline.

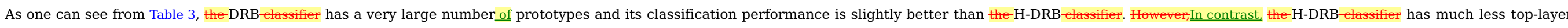

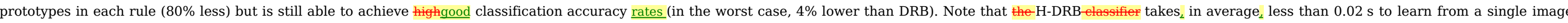

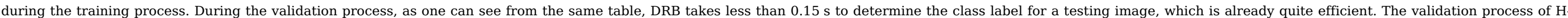

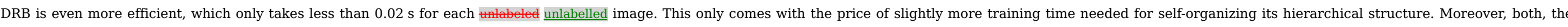

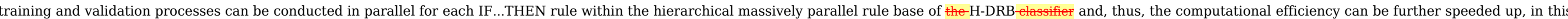

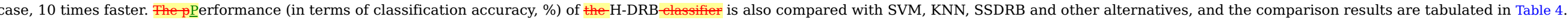

Table 3 Performance of H-DRB and DRB classifiers on MNIST and Fashion MNIST datasets.

\begin{tabular}{|c|c|c|c|c|c|c|}
\hline Dataset & \# Training images & Algorithm & \# Prototypes per class & Accuracy, $\%$ & Training time, $\mathrm{s}$ & Testing time, $\mathrm{s}$ \\
\hline \multirow[t]{7}{*}{ MNIST } & \multirow[t]{2}{*}{5000} & H-DRB & 45.8 & 96.3 & 3.4 & 16.3 \\
\hline & & DRB & 237.5 & 97.1 & 2.5 & 132.0 \\
\hline & \multirow[t]{2}{*}{10000} & H-DRB & 88.4 & 97.0 & 14.5 & 32.8 \\
\hline & & DRB & 463.3 & 97.7 & 12.4 & 270.6 \\
\hline & \multirow[t]{2}{*}{20000} & H-DRB & 139.2 & 97.6 & 80.6 & 102.2 \\
\hline & & DRB & 907.4 & 98.2 & 73.1 & 552.3 \\
\hline & 30000 & $\mathrm{H}-\mathrm{DRB}$ & 242.1 & 97.9 & 201.6 & 147.3 \\
\hline
\end{tabular}




\begin{tabular}{|c|c|c|c|c|c|c|}
\hline & & DRB & 1339.5 & 98.4 & 182.7 & 782.1 \\
\hline & \multirow[t]{2}{*}{40000} & H-DRB & 310.7 & 98.1 & 336.8 & 180.6 \\
\hline & & DRB & 1772.0 & 98.5 & 302.0 & 973.5 \\
\hline & \multirow[t]{2}{*}{50000} & H-DRB & 377.1 & 98.2 & 518.0 & 216.7 \\
\hline & & DRB & 2202.9 & 98.6 & 460.9 & 1217.0 \\
\hline & \multirow[t]{2}{*}{60000} & H-DRB & 437.4 & 98.3 & 749.8 & 240.0 \\
\hline & & DRB & 2631.0 & 98.6 & 662.5 & 1407.9 \\
\hline \multirow[t]{14}{*}{ Fashion MNIST } & \multirow[t]{2}{*}{5000} & H-DRB & 39.9 & 83.8 & 3.4 & 15.3 \\
\hline & & DRB & 230.9 & 84.9 & 2.6 & 131.0 \\
\hline & \multirow[t]{2}{*}{10000} & H-DRB & 76.5 & 85.1 & 12.6 & 24.2 \\
\hline & & DRB & 457.8 & 86.0 & 10.3 & 224.4 \\
\hline & \multirow[t]{2}{*}{20000} & H-DRB & 139.2 & 85.7 & 81.1 & 69.7 \\
\hline & & DRB & 907.4 & 87.0 & 71.0 & 517.8 \\
\hline & \multirow[t]{2}{*}{30000} & H-DRB & 182.7 & 85.8 & 202.1 & 108.8 \\
\hline & & DRB & 1353.9 & 87.6 & 171.6 & 757.5 \\
\hline & \multirow[t]{2}{*}{40000} & H-DRB & 208.4 & 85.4 & 384.7 & 127.2 \\
\hline & & DRB & 1792.9 & 88.0 & 312.4 & 1009.5 \\
\hline & \multirow[t]{2}{*}{50000} & H-DRB & 222.7 & 84.9 & 577.5 & 132.6 \\
\hline & & DRB & 2229.6 & 88.4 & 470.6 & 1219.9 \\
\hline & \multirow[t]{2}{*}{60000} & H-DRB & 232.7 & 84.9 & 836.9 & 135.7 \\
\hline & & DRB & 2662.5 & 88.6 & 673.4 & 1422.4 \\
\hline
\end{tabular}

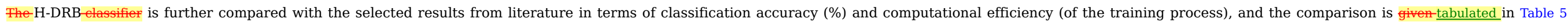
Note that many alternative approaches reported in this table, such as [57,58], actually use GPU for computation.

Table 4 Performance comparison on MNIST and Fashion MNIST datasets with partial training set (the best result is in bold).

\begin{tabular}{|c|c|c|c|c|c|c|c|c|}
\hline Dataset & \# Training images & H-DRB & DRB & SVM & KNN & SSDRB & eClass1 [55] & TEDA Class [56] \\
\hline \multirow[t]{7}{*}{ MNIST } & 5000 & 96.3 & 97.1 & 97.3 & 96.8 & 97.4 & 96.9 & 97.2 \\
\hline & 10000 & 97.0 & 97.7 & 97.9 & 97.5 & 97.8 & 97.2 & 97.4 \\
\hline & 20000 & 97.6 & 98.2 & 98.2 & 98.0 & 98.2 & 97.3 & 97.5 \\
\hline & 30000 & 97.9 & 98.4 & 98.4 & 98.2 & 98.4 & 97.5 & 97.7 \\
\hline & 40000 & 98.1 & 98.5 & 98.5 & 98.3 & 98.5 & 97.5 & 97.7 \\
\hline & 50000 & 98.2 & 98.6 & 98.5 & 98.3 & 98.6 & 97.5 & 97.7 \\
\hline & 60000 & 98.3 & 98.6 & 98.6 & 98.4 & 98.6 & 97.5 & 97.6 \\
\hline Fashion MNIST & 5000 & 83.8 & 84.9 & 86.5 & 84.9 & 85.0 & - & - \\
\hline
\end{tabular}




\begin{tabular}{|l|l|l|l|l|l|l|l|}
\hline 10000 & 85.1 & 86.0 & $\mathbf{8 7 . 7}$ & 86.2 & 86.0 & - \\
\hline 20000 & 85.7 & 87.0 & $\mathbf{8 8 . 6}$ & 87.1 & 87.1 & - & - \\
\hline 30000 & 85.8 & 87.6 & $\mathbf{8 8 . 9}$ & 87.7 & 87.7 & - & - \\
\hline 40000 & 85.4 & 88.0 & $\mathbf{8 9 . 2}$ & 88.1 & 88.0 & - \\
\hline 50000 & 84.9 & 88.4 & $\mathbf{8 9 . 5}$ & 88.4 & 88.4 & - & - \\
\hline 60000 & 84.9 & 88.6 & $\mathbf{8 9 . 4}$ & 88.7 & 88.6 & - \\
\hline
\end{tabular}

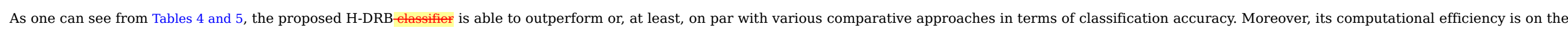

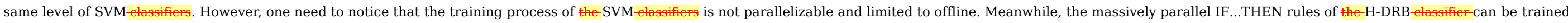
independently and updated with new data samples recursively.

Table 5 Performance comparison on MNIST and Fashion MNIST datasets with the literature (the best result is in bold)

\begin{tabular}{|c|c|c|c|c|}
\hline \multirow[t]{2}{*}{ Algorithm } & \multicolumn{2}{|r|}{ MNIST } & \multicolumn{2}{|c|}{ Fashion MNIST } \\
\hline & Accuracy, \% & Training time & Accuracy, \% & Training time \\
\hline H-DRB & 98.3 & $12 \min , 29 \mathrm{~s}$ & 84.9 & $13 \mathrm{~min}, 57 \mathrm{~s}$ \\
\hline DRB & 98.6 & $11 \mathrm{~min}, 2 \mathrm{~s}$ & 88.6 & $11 \mathrm{~min}, 13 \mathrm{~s}$ \\
\hline Logistic Regression [40] & 90.9 & $26 \mathrm{~h}, 10 \mathrm{~min}, 12 \mathrm{~s}$ & 83.9 & $2 \mathrm{~h}, 59 \mathrm{~min}, 26 \mathrm{~s}$ \\
\hline $\begin{array}{l}\text { Decision tree classifier [40] } \\
\text { Maximum split: } 50\end{array}$ & 88.6 & $2 \mathrm{~min}, 14 \mathrm{~s}$ & 78.9 & $36 \mathrm{~s}$ \\
\hline Passive Aggressive Classifier & 88.0 & $29 \mathrm{~s}$ & 77.3 & $42 \mathrm{~s}$ \\
\hline $\begin{array}{l}\text { Multiple Layer Perception [40] } \\
\text { Actitation Activation function: RELU } \\
\text { Hidden layer size: } 100\end{array}$ & 97.2 & $6 \mathrm{~min}, 55 \mathrm{~s}$ & 87.1 & $16 \min , 3 \mathrm{~s}$ \\
\hline $\begin{array}{l}\text { SVM [40] } \\
\text { Polynomial kernel }\end{array}$ & 97.6 & $1 \mathrm{~h}, 15 \mathrm{~min}, 29 \mathrm{~s}$ & 89.7 & $1 \mathrm{~h}, 12 \min , 39 \mathrm{~s}$ \\
\hline $\begin{array}{l}\text { SVM [40] } \\
\text { Gaussian kernel }\end{array}$ & 97.3 & $48 \mathrm{~min}, 32 \mathrm{~s}$ & 89.7 & $1 \mathrm{~h}, 15 \mathrm{~min}, 25 \mathrm{~s}$ \\
\hline Committee of 7 DCNNs [57] & 99.7 & $\begin{array}{l}98 \mathrm{~h} \\
(14 \mathrm{~h} \text { per DCNN) }\end{array}$ & - & - \\
\hline Committee of 35 DCNNs [58] & 99.8 & $\begin{array}{l}490 \mathrm{~h} \\
(14 \mathrm{~h} \text { per DCNN) }\end{array}$ & - & - \\
\hline Invariant Feature Hierarchies [59] & 99.4 & No Information & - & - \\
\hline Two-Stage Predictive Sparse Decomposition [59] & 99.5 & & - & - \\
\hline DRB Ensemble with GIST Feature [23] & 99.3 & $\sim 2 \mathrm{~h}, 30 \mathrm{~min}$ & - & - \\
\hline DRB Ensemble with HOG Feature [23] & 98.9 & & - & - \\
\hline DRB Ensemble with Combined GIST and HOC Features [23] & 99.3 & & - & - \\
\hline Committee of DRB Ensembles with HOG Feature and with GIST Feature [23] & 99.3 & $\sim 5 \mathrm{~h}$ & - & - \\
\hline DRB Ensemble Cascade [23] & 99.6 & & - & - \\
\hline
\end{tabular}




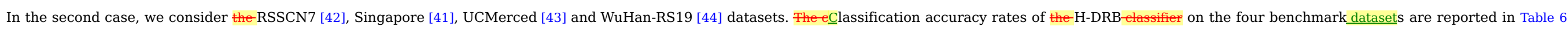

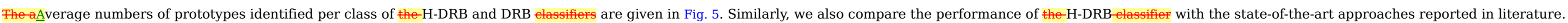

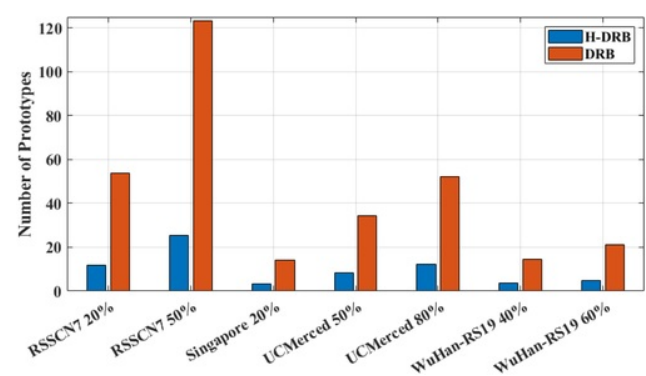

Fig. 5 Average numbers of prototypes identified per class from the remote sensing datasets. The blue bars correspond to the

Table 6 Performance comparison on remote sensing datasets.

\begin{tabular}{|c|c|c|c|c|c|c|c|}
\hline \multirow[t]{2}{*}{ Algorithm } & \multicolumn{2}{|c|}{ RSSCN7 } & \multirow[t]{2}{*}{ Singapore } & \multicolumn{2}{|c|}{ UCMerced } & \multicolumn{2}{|c|}{ Wuhan-RS19 } \\
\hline & $20 \%$ Training & $50 \%$ Training & & $50 \%$ Training & $80 \%$ Training & $40 \%$ Training & $60 \%$ Training \\
\hline H-DRB & $86.2 \pm 0.9$ & $89.8 \pm 0.7$ & $97.1 \pm 0.6$ & $91.8 \pm 0.7$ & $95.5 \pm 1.1$ & $93.1 \pm 1.1$ & $94.1 \pm 0.9$ \\
\hline DRB & $87.2 \pm 0.8$ & $90.8 \pm 0.5$ & $97.3 \pm 0.4$ & $92.9 \pm 0.3$ & $96.7 \pm 1.3$ & $93.3 \pm 0.8$ & $94.7 \pm 0.4$ \\
\hline SVM & $88.7 \pm 0.9$ & $91.1 \pm 0.5$ & $97.7 \pm 0.6$ & $93.3 \pm 0.4$ & $96.8 \pm 1.3$ & $94.9 \pm 1.1$ & $95.9 \pm 0.8$ \\
\hline $\mathrm{KNN}$ & $87.6 \pm 0.8$ & $91.4 \pm 0.5$ & $97.7 \pm 0.4$ & $94.4 \pm 0.6$ & $97.0 \pm 0.9$ & $93.6 \pm 0.8$ & $95.3 \pm 0.7$ \\
\hline SSDRB & $88.0 \pm 0.6$ & $91.1 \pm 0.5$ & $97.8 \pm 0.5$ & $94.1 \pm 0.6$ & $97.8 \pm 1.2$ & $93.4 \pm 1.0$ & $94.9 \pm 0.4$ \\
\hline Gan et al. [41] & - & - & 90.9 & - & 91.1 & - & - \\
\hline Yang \& Newsam [43], [60] & $76.3 \pm 0.9$ & $81.3 \pm 0.6$ & - & $71.9 \pm 0.8$ & $74.1 \pm 3.3$ & $75.3 \pm 1.4$ & $80.1 \pm 2.0$ \\
\hline Hu et al. [49] & - & - & - & - & 98.5 & - & 98.9 \\
\hline Xia et al. [60] & $85.6 \pm 1.0$ & $88.9 \pm 0.6$ & & $94.1 \pm 1.0$ & $95.2 \pm 1.2$ & $95.1 \pm 1.2$ & $96.2 \pm 0.6$ \\
\hline Wu et al. [61] & - & $90.4 \pm 0.6$ & - & - & $92.7 \pm 0.8$ & - & - \\
\hline Wu et al. [62] & - & $86.4 \pm 0.7$ & - & - & $91.8 \pm 1.3$ & - & - \\
\hline Zhao et al. [63] & - & 89.1 & - & - & 97.8 & - & - \\
\hline Lazebnik et al. $[60,64]$ & $68.9 \pm 0.7$ & $72.9 \pm 0.9$ & - & $58.3 \pm 1.9$ & $62.4 \pm 1.9$ & $54.4 \pm 2.2$ & $58.5 \pm 2.3$ \\
\hline Jegou et al. $[60,65]$ & $77.3 \pm 0.6$ & $82.3 \pm 1.2$ & - & $73.2 \pm 1.0$ & $78.2 \pm 1.7$ & $76.4 \pm 2.0$ & $80.8 \pm 2.2$ \\
\hline Bian et al. [66] & - & - & - & $94.2 \pm 1.0$ & $95.8 \pm 1.0$ & $95.4 \pm 0.8$ & $96.4 \pm 0.8$ \\
\hline Huang et al. [67] & - & - & - & - & $93.0 \pm 1.2$ & - & $94.3 \pm 1.0$ \\
\hline Chen et al. [68] & - & - & - & - & $90.0 \pm 2.1$ & - & $91.0 \pm 1.5$ \\
\hline Qi et al. [69] & - & - & - & - & $91.1 \pm 0.7$ & - & $91.7 \pm 1.1$ \\
\hline Nogueira et al. [70] & - & - & - & - & $99.5 \pm 0.5$ & - & $94.5 \pm 1.2$ \\
\hline Chaib et al. [71] & - & - & - & - & $97.4 \pm 1.8$ & - & $98.7 \pm 0.2$ \\
\hline Wang et al. [72] & - & - & - & $96.8 \pm 0.1$ & $99.1 \pm 0.4$ & $97.5 \pm 0.5$ & $99.8 \pm 0.3$ \\
\hline
\end{tabular}




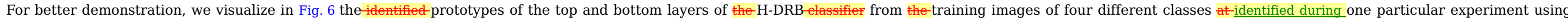

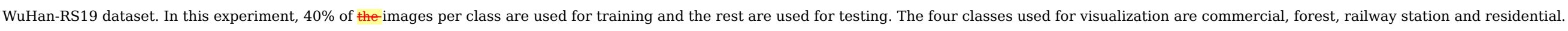

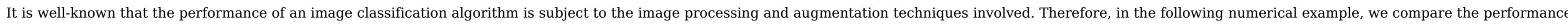

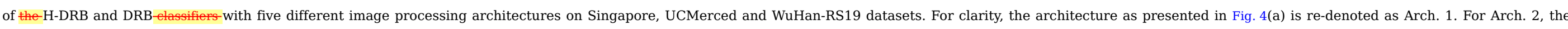

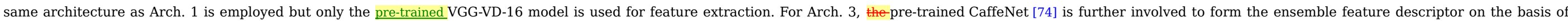

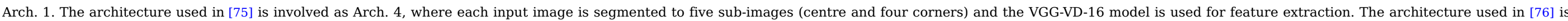

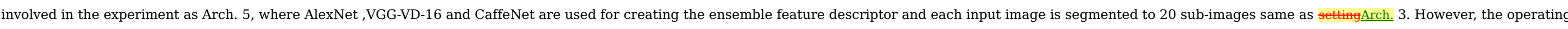

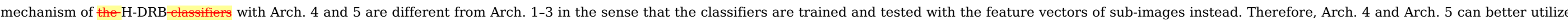

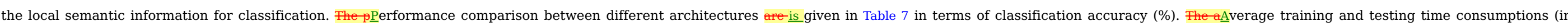
seconds) of the H-DRB and DRB-classifiers with the respective five architectures across the numerical experiments are reported in the same table.
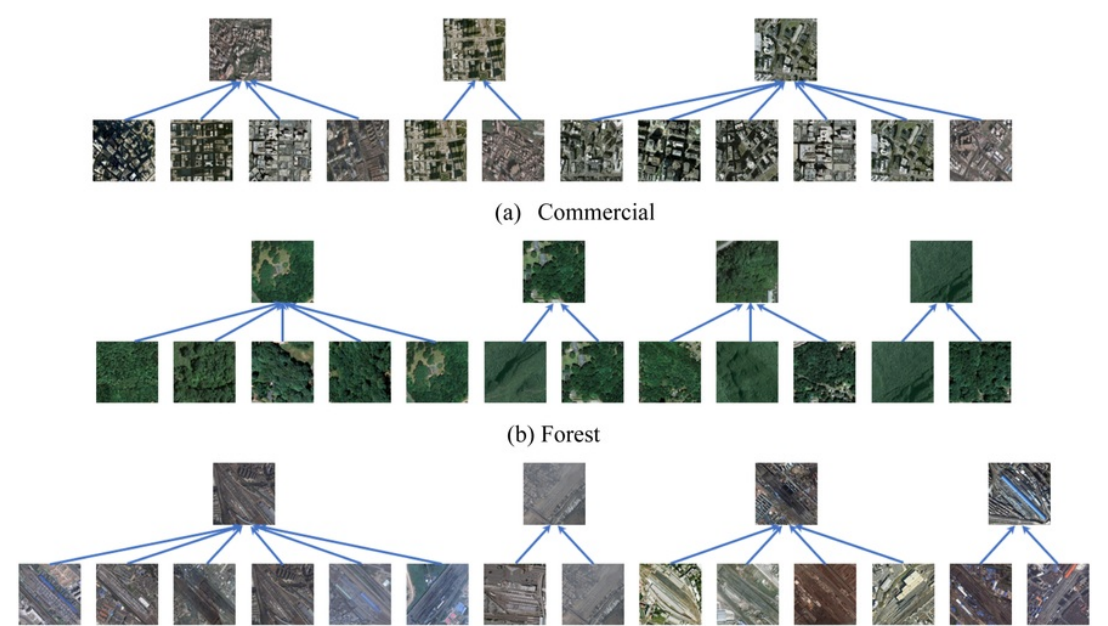

(c) Railway statio

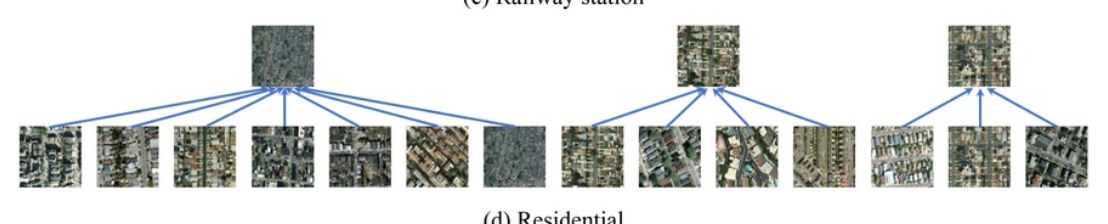

(d) Residential

Fig. 6 Example of identified prototypes at the bottom and top layers of the-H-DRB-classifior.

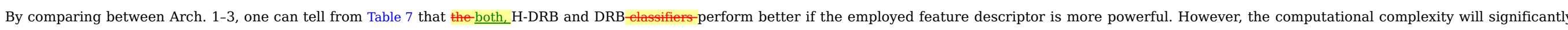

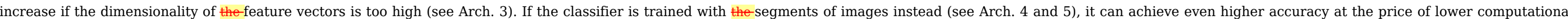
efficiency.

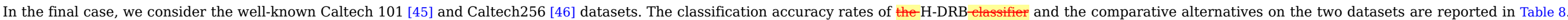
The anverage numbers of prototypes that the-H-DRB-elassifier identifies form the training images of each class are given in Fig. 7 and compared with the-DRB-elassifier. 
Table 7 Performance comparison of H-DRB and DRB with different architectures.

\begin{tabular}{|c|c|c|c|c|c|c|c|c|}
\hline \multicolumn{2}{|c|}{ Algorithm } & \multicolumn{5}{|c|}{ Accuracy, \% } & \multicolumn{2}{|c|}{ Time Consumption, s } \\
\hline & & \multirow[t]{2}{*}{ Singapore } & \multicolumn{2}{|c|}{ UCMerced } & \multicolumn{2}{|c|}{ WuHan-RS19 } & \multirow[t]{2}{*}{ Training } & \multirow[t]{2}{*}{ Testing } \\
\hline & & & $50 \%$ & $80 \%$ & $40 \%$ & $60 \%$ & & \\
\hline \multirow[t]{2}{*}{ Arch. 1} & H-DRB & 97.1 & 91.8 & 95.5 & 93.1 & 94.1 & 1.8 & 2.5 \\
\hline & DRB & 97.3 & 92.9 & 96.7 & 93.3 & 94.7 & 1.5 & 12.9 \\
\hline \multirow[t]{2}{*}{ Arch. 2} & H-DRB & 92.4 & 88.9 & 93.3 & 90.0 & 90.8 & 0.9 & 1.6 \\
\hline & DRB & 95.6 & 91.6 & 96.1 & 91.4 & 93.0 & 0.7 & 6.6 \\
\hline \multirow[t]{2}{*}{ Arch. 3} & H-DRB & 96.5 & 92.8 & 96.5 & 93.7 & 94.7 & 3.5 & 4.9 \\
\hline & DRB & 96.7 & 93.7 & 97.4 & 94.2 & 95.1 & 2.8 & 22.6 \\
\hline \multirow[t]{2}{*}{ Arch. 4} & H-DRB & 97.3 & 90.2 & 94.5 & 93.7 & 94.4 & 10.0 & 21.3 \\
\hline & DRB & 97.8 & 90.9 & 95.8 & 93.0 & 93.5 & 9.8 & 97.8 \\
\hline \multirow[t]{2}{*}{ Arch. 5} & H-DRB & 97.8 & 93.6 & 97.4 & 94.9 & 96.2 & 653.0 & 1743.9 \\
\hline & DRB & 97.8 & 94.0 & 97.3 & 95.2 & 96.3 & 617.0 & 7424.3 \\
\hline
\end{tabular}

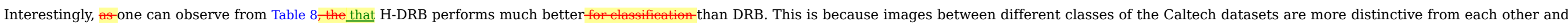

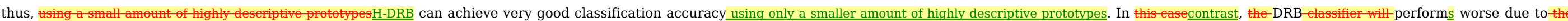
overfitting.

Table 8 Numerical results on Galtech101 datasetCaltech datasets.

\begin{tabular}{|c|c|c|c|c|c|c|}
\hline \multirow[t]{2}{*}{ Algorithm } & \multicolumn{2}{|c|}{ Caltech101 } & \multicolumn{4}{|c|}{ Caltech256 } \\
\hline & 15 & 30 & 15 & 30 & 45 & 60 \\
\hline H-DRB & $86.7 \pm 0.7$ & $89.5 \pm 0.6$ & $64.7 \pm 0.4$ & $68.9 \pm 0.3$ & $71.2 \pm 0.3$ & $73.1 \pm 0.3$ \\
\hline $\mathrm{DRB}$ & $84.9 \pm 0.6$ & $88.6 \pm 0.5$ & $62.4 \pm 0.3$ & $67.1 \pm 0.3$ & $69.8 \pm 0.3$ & $71.9 \pm 0.3$ \\
\hline SVM & $87.3 \pm 1.0$ & $90.3 \pm 0.9$ & \multicolumn{4}{|c|}{ Out of System Memory } \\
\hline KNN & $86.7 \pm 0.6$ & $90.0 \pm 0.5$ & $62.5 \pm 0.3$ & $67.2 \pm 0.3$ & $69.9 \pm 0.3$ & $72.1 \pm 0.3$ \\
\hline SSDRB & $85.5 \pm 0.9$ & $89.2 \pm 0.7$ & $64.1 \pm 0.4$ & $68.0 \pm 0.2$ & $70.4 \pm 0.3$ & $72.3 \pm 0.3$ \\
\hline Gao et al. [5] & $71.3 \pm 0.6$ & $77.6 \pm 1.0$ & $35.1 \pm 0.4$ & $42.1 \pm 0.3$ & $46.0 \pm 0.3$ & $48.5 \pm 0.3$ \\
\hline Xie et al. [77] & 76.0 & 82.5 & 36.4 & 45.1 & 48.0 & 50.3 \\
\hline Li et al. [78] & - & 89.2 & - & - & - & 74.9 \\
\hline Wang et al. [79] & $64.0 \pm 0.4$ & $71.4 \pm 1.2$ & - & - & - & - \\
\hline Yang et al. [80] & $67.0 \pm 0.5$ & $73.2 \pm 0.5$ & $27.7 \pm 0.5$ & $34.0 \pm 0.4$ & $37.5 \pm 0.6$ & $40.1 \pm 0.9$ \\
\hline Saban et al. [81] & 68.5 & 75.0 & - & - & - & - \\
\hline Pan et al. [82] & $77.2 \pm 0.6$ & $85.8 \pm 0.4$ & $36.6 \pm 0.6$ & $47.2 \pm 0.7$ & $50.8 \pm 0.4$ & $52.9 \pm 0.5$ \\
\hline Zhang et al. [83] & - & - & $61.5 \pm 0.4$ & $67.7 \pm 0.7$ & $69.8 \pm 0.5$ & $72.8 \pm 0.4$ \\
\hline
\end{tabular}




\subsection{Discussions}

Based on the numerical examples presented in this section, the following four remarks are worth noting

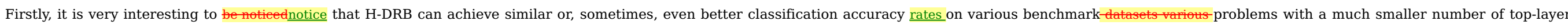

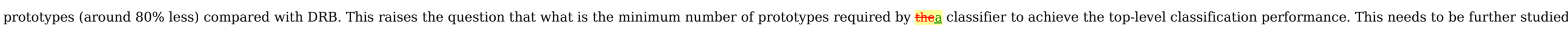
in order to fully understand the advantages and limitations of prototype-based classifiers.

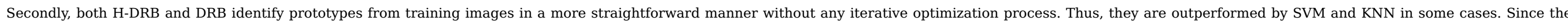
optimality of prototypes determine the performance of the prototype-based approaches [48], one may need to introduce a prototype optimization mechanism to H-DRB and DRB to maximize their strength.

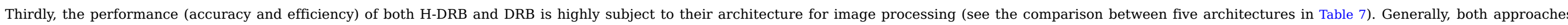

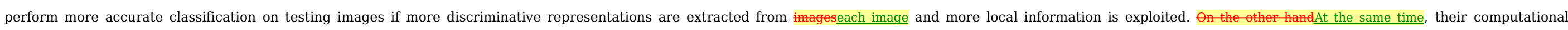

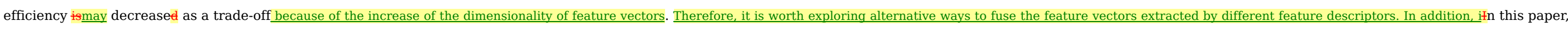

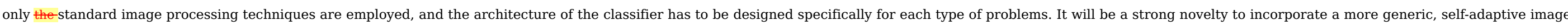
processing architecture for the

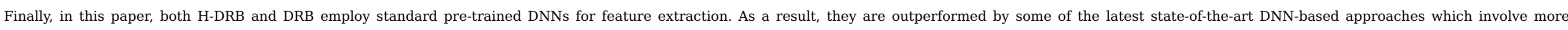

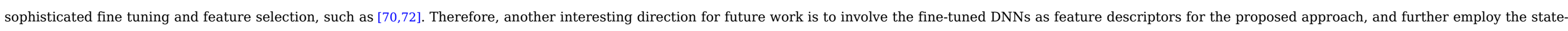
of-the-art feature selection techniques for dimensionality reduction.

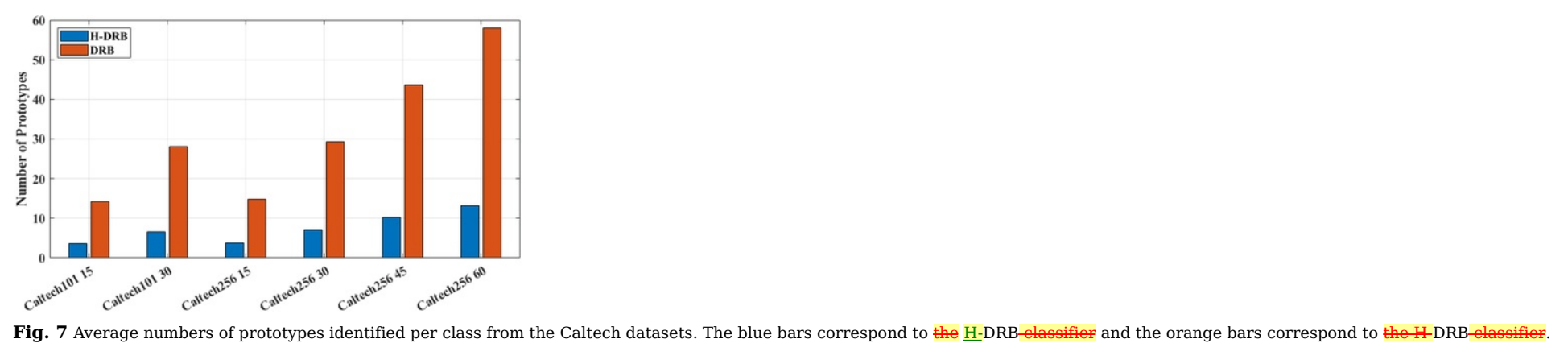

\section{Conclusion}

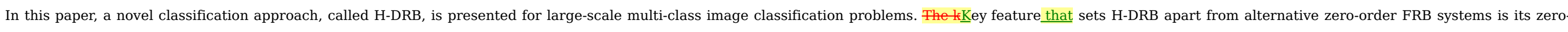

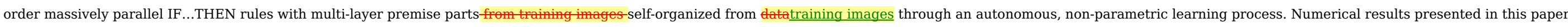

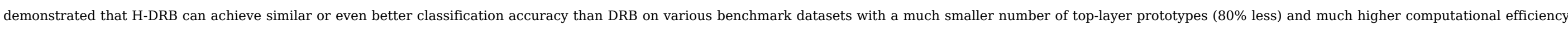

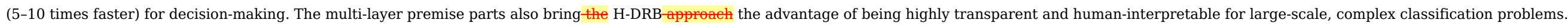

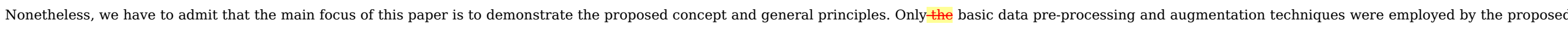

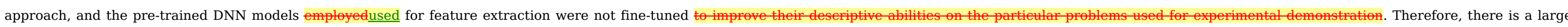

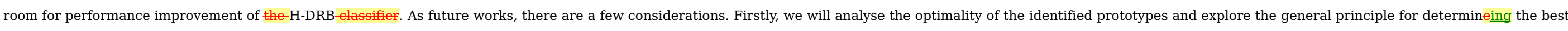

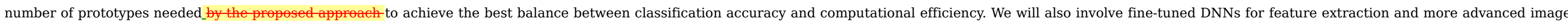




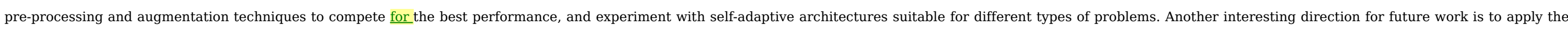
proposed approach to video classification by further taking the time and space correlation into consideration.

\section{Declaration of Competing Interest}

The authors declare that they have no known competing financial interests or personal relationships that could have appeared to influence the work reported in this paper.

\section{References}

[1] Y. LeCun, Y. Bengio and G. Hinton, Deep learning, Nature Methods 13 (1), 2015, 35.

[2] V. Mnih, et al., Human-level control through deep reinforcement learning, Nature 518 (7540), 2015, 529-533.

[3] R. Shwartz-Ziv and N. Tishby, Opening the black box of deep neural networks via information, 2017, arXiv Prepr. arXiv:1703.00810.

[4] K. He, X. Zhang, S. Ren, J. Sun, Deep residual learning for image recognition, in: IEEE Conference on Computer Vision and Pattern Recognition (CVPR), 2016 , pp. 770-778.

[5] S. Gao, L. Duan and I.W. Tsang, DEFEATnet-a deep conventional image representation for image classification, IEEE Trans. Circuits Syst. Video Technol. 26 (3), 2016 , 494-505.

[6] G. Hinton, et al., Deep neural networks for acoustic modeling in speech recognition, IEEE Signal Process. Mag. 29, 2012, 82-97.

[7] D. Amodei, et al. Deep speech 2: End-to-end speech recognition in english and mandarin, in: International conference on machine learning, 2016 , pp. 173-182.

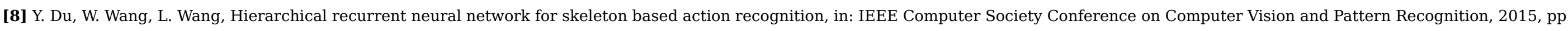
1110-1118.

[9] Y. Li, H. Zhang, X. Xue, Y. Jiang and Q. Shen, Deep learning for remote sensing image classification: A survey, Wiley Interdiscip. Rev. Data Min. Knowl. Discov. e1264, 2018.

[10] J.M. Garibaldi, The need for fuzzy AI, IEEE/CAA J. Autom. Sin. 6 (3), 2019, 610-622.

[11] H. Hagras, Toward human-understandable, explainable AI, Computer (Long. Beach. Calif). 51 (9), 2018, 28-36.

[12] X. Ding, Y. Zhang, T. Liu, J. Duan, Deep learning for event-driven stock prediction, in: International Joint Conference on Artificial Intelligence, 2015 , pp. $2327-2333$.

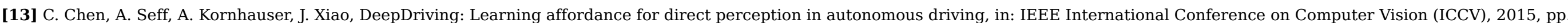
2722-2730.

[14] J. Su, D.V. Vargas and K. Sakurai, One pixel attack for fooling deep neural networks, IEEE Trans. Evol. Comput. 2019, https://doi.org/10.1109/TEVC.2019.2890858.

[15] P.P. Angelov and X. Gu, Toward anthropomorphic machine learning, IEEE Comput. Mag. 51 (9), 2018, 18-27.

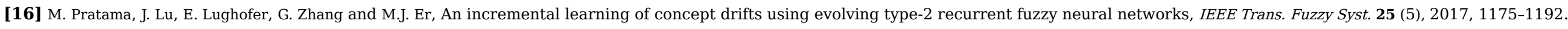

[17] N.K. Kasabov and Q. Song, DENFIS: Dynamic evolving neural-fuzzy inference system and its application for time-series prediction, IEEE Trans. Fuzzy Syst. 10 (2), 2002, 144-154.

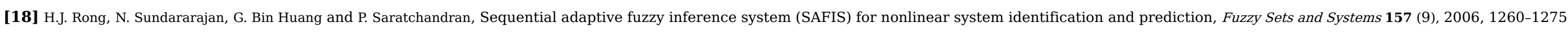

[19] M. Pratama, S.G. Anavatti, P.P. Angelov and E. Lughofer, PANFIS: A novel incremental learning machine, IEEE Trans. Neural Netw. Learn. Syst. 25 (1), 2014 , 55-68.

[20] J. Soto, P. Melin and O. Castillo, A new approach for time series prediction using ensembles of IT2FNN models with optimization of fuzzy integrators, Int. J. Fuzzy Syst. 20 (3), 2018, 701-728.

[21] I. Ben Ali, M. Turki, J. Belhadj and X. Roboam, Optimized fuzzy rule-based energy management for a battery-less PV/wind-BWRO desalination system, Energy 159, 2018, 216-228.

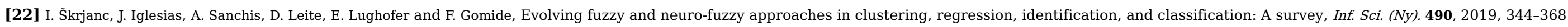


[23] P.P. Angelov and X. Gu, Deep rule-based classifier with human-level performance and characteristics, Inf. Sci. (Ny). 463-464, 2018, 196-213.

[24] L. Perez and J. Wang, The effectiveness of data augmentation in image classification using deep learning, 2017, arXiv Prepr. arXiv:1712.

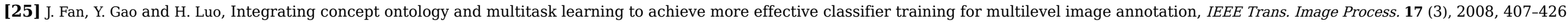

[26] M. Marszałek, C. Schmid, Constructing category hierarchies for visual recognition, in: European Conference on Computer Vision, 2008 , pp. 479-491.

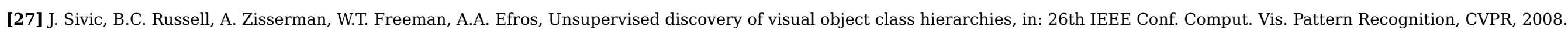

[28] J. Fan, et al., HD-MTL: Hierarchical deep multi-task learning for large-scale visual recognition, IEEE Trans. Image Process. 26 (4), 2017, 1923-1938.

[29] Y. Qu, et al., Joint hierarchical category structure learning and large-scale image classification, IEEE Trans. Image Process. 26 (9), 2017, 4331-4346.

[30] T. Zhao, et al., Embedding visual hierarchy with deep networks for large-scale visual recognition, IEEE Trans. Image Process. 27 (10), $2018,4740-4755$.

[31] J. Fan, N. Zhou, J. Peng and L. Gao, Hierarchical learning of tree classifiers for large-scale plant species identification, IEEE Trans. Image Process. 24 (11), 2015, 4172-4184.

[32] X. Gu and W. Ding, A hierarchical prototype-based approach for classification, Inf. Sci. (Ny). 505, 2019, 325-351.

[33] D.G. Lowe, Distinctive image features from scale-invariant keypoints, Int. J. Comput. Vis. 60 (2), 2004, 91-110.

[34] A. Krizhevsky, I. Sutskever and G.E. Hinton, Imagenet classification with deep convolutional neural networks, In: Advances in Neural Information Processing Systems, 2012, $1097-1105$.

[35] K. Simonyan, A. Zisserman, Very deep convolutional networks for large-scale image recognition, in: International Conference on Learning Representations, 2015, pp. 1-14.

[36] C. Szegedy, et al., Going deeper with convolutions, In: IEEE Conference on Computer Vision and Pattern Recognition, 2015, 1-9.

[37] C.C. Aggarwal and C.K. Reddy, (Eds.), Data Clustering: Algorithms and Applications, 2013, CRC press.

[38] X. Gu, P.P. Angelov and J.C. Principe, A method for autonomous data partitioning, Inf. Sci. (Ny) 460-461, 2018, 65-82.

[39] Y. LeCun, L. Bottou, Y. Bengio and P. Haffner, Gradient-based learning applied to document recognition, Proc. IEEE 86 (11), 1998, $2278-2323$.

[40] H. Xiao, K. Rasul and R. Vollgraf, Fashion-MNIST: A novel image dataset for benchmarking machine learning algorithms, 2017, arXiv Prepr. arXiv:1708.07747.

[41] J. Gan, Q. Li, Z. Zhang and J. Wang, Two-level feature representation for aerial scene classification, IEEE Geosci. Remote Sens. Lett. 13 (11), 2016 , 1626-1630.

[42] Q. Zou, L. Ni, T. Zhang and Q. Wang, Deep learning based feature selection for remote sensing scene classification, IEEE Geosci. Remote Sens. Lett. 12 (11), 2015, 2321-2325.

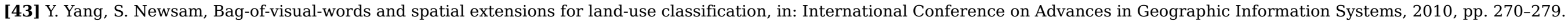

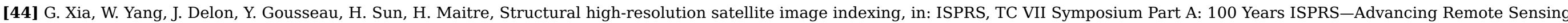
Science, 2010, pp. 298-303.

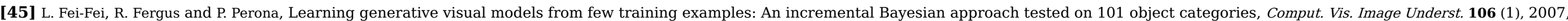
59-70.

[46] G. Griffin, A. Holub and P. Perona, Caltech-256 Object Category Dataset, 2017.

[47] A. Oliva and A. Torralba, Modeling the shape of the scene: A holistic representation of the spatial envelope, Int. J. Comput. Vis. 42 (3), 2001, 145-175.

[48] X. Gu, P. Angelov and H.J. Rong, Local optimality of self-organising neuro-fuzzy inference systems, Inf. Sci. (Ny). 503, 2019, 351-380.

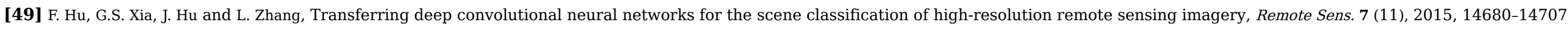


[50] N. Cristianini and J. Shawe-Taylor, An Introduction to Support Vector Machines and Other Kernel-Based Learning Methods, 2000, Cambridge University Press; Cambridge.

[51] P. Cunningham and S.J. Delany, K-nearest neighbour classifiers, Mult. Classif. Syst. 34, 2007, 1-17.

[52] X. Gu and P.P. Angelov, Semi-supervised deep rule-based approach for image classification, Appl. Soft Comput. 68, 2018, 53-68.

[53] M.D. Zeiler, R. Fergus, Visualizing and understanding convolutional networks, in: European Conference on Computer Vsion, 2014 , pp. 818-833.

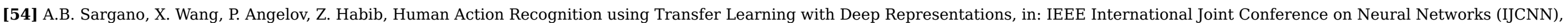
2017, pp. 463-469.

[55] P. Angelov and X. Zhou, Evolving fuzzy-rule based classifiers from data streams, IEEE Trans. Fuzzy Syst. 16 (6), 2008, 1462-1474.

[56] D. Kangin, P. Angelov and J.A. Iglesias, Autonomously evolving classifier TEDAClass, Inf. Sci. (Ny). 366, 2016, 1-11.

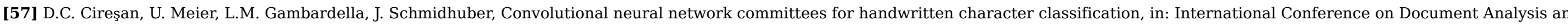
Recognition, vol.10, 2011, pp. 1135-1139.

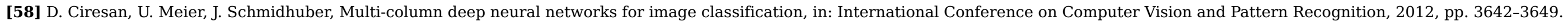

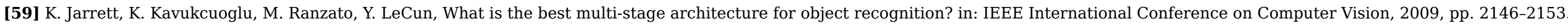

[60] G. Xia, et al., AID: A benchmark dataset for performance evaluation of aerial scene classification, IEEE Trans. Geosci. Remote Sens. 55 (7), 2017 , $3965-3981$.

[61] H. Wu, B. Liu, W. Su, W. Zhang and J. Sun, Deep filter banks for land-use, IEEE Geosci. Remote Sens. Lett. 13 (12), 2016, 1895-1899.

[62] H. Wu, B. Liu, W. Su, W. Zhang and J. Sun, Hierarchical coding vectors for scene level land-use classification, Remote Sens. 8 (5), $2016,436$.

[63] F. Zhao, X. Mu, Z. Yang and Z. Yi, A novel two-stage scene classification model based on feature variable significance in high- resolution remote sensing, Geocarto Int. 2019 , 1-12.

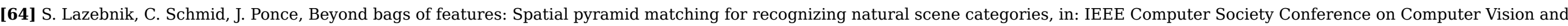
Pattern Recognition, 2006, pp. 2169-2178.

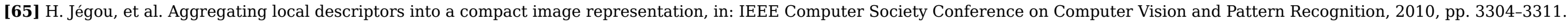

[66] X. Bian, C. Chen, L. Tian and Q. Du, Fusing local and global features for high-resolution scene classification, IEEE J. Sel. Top. Appl. Earth Obs. Remote Sens. 10 (6), 2017, $2889-2901$.

[67] L. Huang, C. Chen, W. Li and Q. Du, Remote sensing image scene classification using multi-scale completed local binary patterns and fisher vectors, Remote Sens. 8 (6), 2016, 1-17.

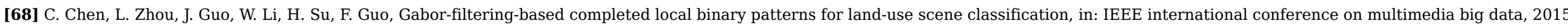
pp. 324-329.

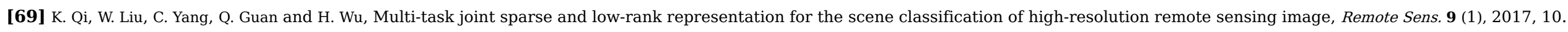

[70] K. Nogueira, O.A.B. Penatti and J.A. dos Santos, Towards better exploiting convolutional neural networks for remote sensing scene classification, Pattern Recognit. 61, 2017 , 539-556.

[71] S. Chaib, H. Liu, Y. Gu and H. Yao, Deep feature fusion for VHR remote sensing scene classification, IEEE Trans. Geosci. Remote Sens. 55 (8), 2017 , $4775-4784$.

[72] Q. Wang, S. Member, S. Liu and J. Chanussot, Scene classification with recurrent attention of VHR remote sensing images, IEEE Trans. Geosci. Remote Sens. 57 (2), 2019, 1155-1167.

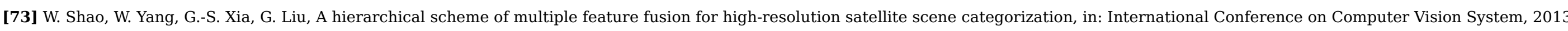
pp. 324-333. 
[74] Y. Jia, et al. Caffe: Convolutional architecture for fast feature embedding * , in: ACM International Conference on Multimedia, 2014, pp. 675-678.

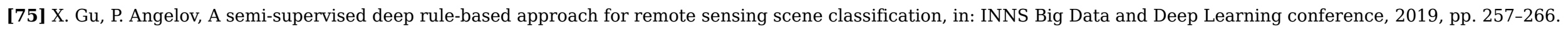

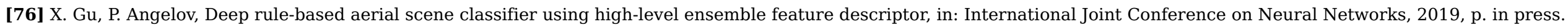

[77] L. Xie, Q. Tian, M. Wang and B. Zhang, Spatial pooling of heterogeneous features for image classification, IEEE Trans. Image Process. 23 (5), $2014,1994-2008$.

[78] Q. Li, Q. Peng and C. Yan, Multiple VLAD encoding of CNNs for image classification, Comput. Sci. Eng. 20 (2), 2018, 52-63.

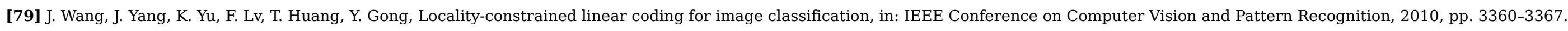

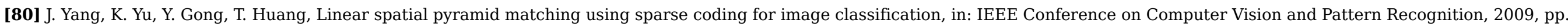
1794-1801.

[81] A. Shaban, H.R. Rabiee, M. Najibi and S. Yousefi, From local similarities to global coding: A framework for coding applications, IEEE Trans. Image Process. 24 (12), 2015, 5074-5085.

[82] Y. Pan, Y. Xia, Y. Song and W. Cai, Locality constrained encoding of frequency and spatial information for image classification, Multimed. Tools Appl. 77 (19), $2018,24891-24907$.

[83] C. Zhang, J. Cheng and Q. Tian, Structured weak semantic space construction for visual categorization, IEEE Trans. Neural Netw. Learn. Syst. 29 (8), $2018,3442-3451$.

[84] C. Zhang, C. Li, D. Lu, J. Cheng and Q. Tian, Birds of a feather flock together: Visual representation with scale and class consistency, Inf. Sci. (Ny). 460-461, $2018,115-127$.

\section{Highlights}

- A generic approach for deep rule-based systems to self-organize a multi-layer structure is proposed.

- The proposed system can offer higher transparency and human-interpretability for large-scale, complex problems.

- The proposed approach can perform highly efficient decision-making with attractive classification precision.

- The effectiveness and validity of the proposed approach are demonstrated on a variety of popular benchmark image tatasets.

\section{Queries and Answers}

Query: Please check whether the designated corresponding author is correct, and amend if necessary.

Answer: Yes, correct.

Query: Please check the corresponding address, and correct if necessary.

Answer: Correct

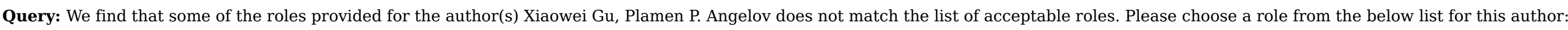

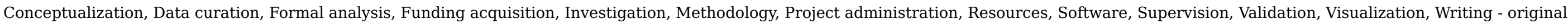
draft, Writing - review $\backslash \&$ editing.

Answer: Xiaowei Gu: methodology, writing-original draft, writing-review $\backslash \&$ editing. $\quad$ Plamen P. Angelov: conceptualization, writing-original draft, writing-review $\backslash \&$ editing.

Query: Please check whether the third affiliation given here is okay. 


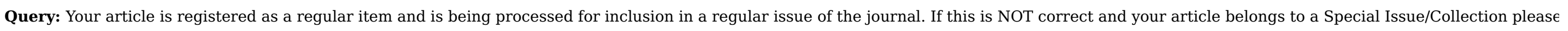
contact immediately prior to returning your corrections.

Answer: Correct.

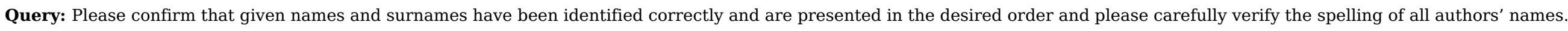
Answer: Yes

Query: Blank spaces have been changed to en dashes in Tables 4, 5, 6, 7 and 8. Please check.

Answer: Correct

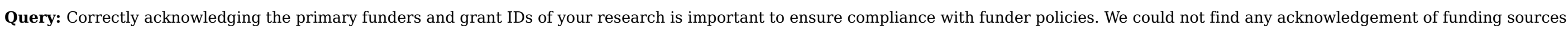
in your text. Is this correct?

Answer: Yes, correct.

Query: Please update the status of publication for Ref. [76].

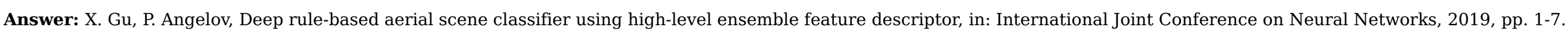

